\title{
Variational eigenvalue approximation of non-coercive operators with application to mixed formulations in elasticity
}

\author{
Salim Meddahi ${ }^{1}$ (1) \\ Received: 14 May 2021 / Accepted: 23 November 2021 / Published online: 4 January 2022 \\ (c) The Author(s) 2021
}

\begin{abstract}
We present an abstract framework for the eigenvalue approximation of a class of noncoercive operators. We provide sufficient conditions to guarantee the spectral correctness of the Galerkin scheme and to obtain optimal rates of convergence. The theory is applied to the convergence analysis of mixed finite element approximations of the elasticity and Stokes eigensystems.
\end{abstract}

Keywords Elasticity eigenproblem $\cdot$ Mixed finite elements $\cdot$ Spectral analysis $\cdot$ Error estimates

Mathematics Subject Classification $65 \mathrm{~N} 30 \cdot 65 \mathrm{~N} 12 \cdot 65 \mathrm{M} 15 \cdot 65 \mathrm{~N} 25$

\section{Introduction}

In many common applications of solid mechanics, mixed formulations derived from the Hellinger-Reissner variational principle perform better than the standard displacement-based formulation. They deliver direct and accurate approximations of the stress tensor and they are free from the locking phenomenon in the nearly incompressible case [11].

The symmetry constraint on the Cauchy stress tensor has been the main difficulty in the construction of stable conforming discretizations of stress-displacement mixed formulations. The first important progress in this direction is due to Arnold and Winther [3]. This work led to further developments in conforming mixed finite elements on simplicial and rectangular meshes for both $2 \mathrm{D}$ and $3 \mathrm{D}$; see $[1,6,23]$ and the references therein. However, these mixed finite elements require the simultaneous imposition of $\mathrm{H}$ (div)-conformity and strong symmetry, which entails too many degrees of freedom and complicates the implementation of the corresponding Galerkin schemes. Moreover, they are not amenable to hybridization. To overcome this difficulty one can either consider non-conforming or DG approximations

Dedicated to the memory of Francisco-Javier Sayas.

Salim Meddahi

salim@uniovi.es

1 Departamento de Matemáticas, Facultad de Ciencias, Universidad de Oviedo, Calvo Sotelo s/n, Oviedo, Spain 
$[7,20,32]$ or relax the symmetry constraint as in $[2,4,5,14,21,31]$. We point out that the latter alternative, which is the option of choice in this paper, incorporates a further variable (a Lagrange multiplier called the rotor that approximates the skew-symmetric part of the displacement gradient) to enforce weakly the symmetry restriction at the discrete level.

The approximation of eigenvalue problems in mixed form has been the object of several papers; see part 3 of [8] and the references therein. In particular, it is known from [9] that the usual stability conditions for discrete mixed source problems (namely the 'coercivity in the kernel' and the inf-sup conditions) are not sufficient to ensure correct spectral approximations. Recently, a dual-mixed eigenvalue formulation of the elasticity problem with reduced symmetry has been considered in [26]. The eigenproblem resulting from this approach doesn't fit into any of the previously existing theories for mixed spectral problems. Nevertheless, the abstract spectral approximation theory of Descloux-Nassif-Rappaz [16] could be successfully adapted in [26] to show that the Galerkin method based on the first order Arnold-Falk-Winther element [5] is free from spurious modes and converges at optimal rates for eigenvalues and eigenvectors. The same strategy has been applied to a pseudostress formulation of the Stokes eigenproblem [28] and to a stress-pressure formulation of a fluid-structure interaction spectral problem [27].

The aim of this paper is to provide a general theory for the spectral approximation of a class of symmetric and noncoercive operators, so that the studies carried out in [26-28] all fit into the same framework. The analysis given here is performed according to the ideas in [26] and builds on the theory developed in $[16,17]$. The resulting unified approach reveals a new criterion (see Assumption 5 below) to determine the spectral correctness of a given Galerkin approximation. This allows to validate more families of mixed finite elements for the approximation of the elasticity eigenproblem as mentioned in Remark 6.1.

We also highlight that the analysis considered in [26] relies on the regularity of an auxiliary elasticity source problem. Here, we can circumvent the use of this property, which allows us to treat the important case of heterogeneous material coefficients.

The paper is organized as follows: In Sect. 2 we set out the abstract spectral problem and we describe its continuous Galerkin approximation in Sect. 3. In Sect. 4 we provide sufficient conditions ensuring the spectral correctness of the approximation in the sense of [16]. In Sect. 5, we establish rates of convergence for eigenvalues and eigenfunctions. Section 6 is devoted to applications. We show that the abstract framework can be applied to the stress formulation with weak symmetry of the elasticity and Stokes eigenproblems. We present numerical results for the latter example that confirm the theoretical convergence rates.

\section{An abstract eigenproblem}

Let $H, X$ be two infinite-dimensional, separable, real Hilbert spaces endowed with inner products $(\cdot, \cdot)_{H},(\cdot, \cdot)_{X}$ and corresponding norms $\|\cdot\|_{H}$ and $\|\cdot\|_{X}$. We assume that the inclusion $X \hookrightarrow H$ is continuous. We let $c: X \times X \rightarrow \mathbb{R}$ be a bounded, symmetric and positive semidefinite bilinear form such that $c(\cdot, \cdot)+(\cdot, \cdot)_{H}$ is coercive on $X$, i.e., there exists $\alpha>0$ such that

$$
c(v, v)+\|v\|_{H}^{2} \geq \alpha\|v\|_{X}^{2}, \quad \forall v \in X .
$$

We introduce the closed subspace

$$
K:=\{u \in X: c(u, v)=0, \quad \forall v \in X\},
$$


and point out that, as $c(\cdot, \cdot)$ is semidefinite, we may also write $K=\{u \in X: c(u, u)=0\}$. We do not require $K$ to be finite-dimensional. Finally, we let $B: H \times H \rightarrow \mathbb{R}$ be a symmetric and bounded bilinear form and consider $A: X \times X \rightarrow \mathbb{R}$ given by

$$
A(u, v):=c(u, v)+B(u, v) .
$$

We are interested in the following eigenvalue problem: find $0 \neq u \in X$ and $\kappa \in \mathbb{R}$ such that

$$
A(u, v)=\kappa B(u, v), \quad \forall v \in X .
$$

Our purpose is to introduce a series of assumptions that permit us to solve (in Sect. 2) the spectral problem (2.1) and to analyse (in Sect. 3) the convergence of the corresponding Galerkin approximation (3.1).

Assumption 1 We assume that

(i) there exists $\beta_{A}>0$ such that $\sup _{v \in X} \frac{A(u, v)}{\|v\|_{X}} \geq \beta_{A}\|u\|_{X}, \quad \forall u \in X$,

(ii) and there exists $\beta_{B}>0$ such that $\sup _{v \in K} \frac{B(u, v)}{\|v\|_{H}} \geq \beta_{B}\|u\|_{H}, \quad \forall u \in K$.

Assumption 1(i) and the symmetry of $A$ imply that the linear operator $T: X \rightarrow X$ defined, for all $u \in X$, by

$$
A(T u, v)=B(u, v), \quad \forall v \in X,
$$

is well-defined and bounded, c.f. [18, Theorem 2.6]. The importance of the source operator $T$ lies in the fact that its eigenvalues and those of the problem (2.1) are reciprocal to each other with coincident associated eigenfunctions. A full description of the spectrum of $T$ will then solve eigenproblem (2.1). It is clear that $\kappa=1$ is an eigenvalue of (2.1) associated with the eigenspace $K$, which can also be expressed in terms of the source operator $T$ by the property $\operatorname{ker}(I-T)=K$. Consequently, if $K$ is not a finite-dimensional subspace of $X$ (which is the case in the applications we have in mind) $T$ is not a compact operator.

We introduce the closed subspace

$$
K^{\perp_{B}}:=\{u \in X: B(u, v)=0, \quad \forall v \in K\} .
$$

We point out that the orthogonality symbol $\perp_{B}$ is an abuse of notation since $B(\cdot, \cdot)$ is generally not an inner product in $H$. Moreover, the symmetry of $A$ and $B$ imply that $T$ is symmetric with respect to $B$, indeed,

$$
B(T u, v)=B(v, T u)=A(T v, T u)=A(T u, T v)=B(u, T v), \quad \forall u, v \in X .
$$

It follows immediately from this fact that $K^{\perp_{B}}$ is $T$-invariant, namely, $T\left(K^{\perp_{B}}\right) \subset K^{\perp_{B}}$.

Proposition 2.1 If Assumption 1 (ii) is satisfied, the splitting $X=K \oplus K^{\perp_{B}}$ is direct and stable.

Proof By virtue of Assumption 1 (ii), for any $u \in X$, there exists a unique $u_{0} \in K$ solution of

$$
B\left(u_{0}, v\right)=B(u, v), \quad \forall v \in K,
$$


with the a priori estimate (see [18, Theorem 2.6]) $\left\|u_{0}\right\|_{X} \leq \frac{\left\|u_{0}\right\|_{H}}{\sqrt{\alpha}} \leq \frac{\|B\|}{\sqrt{\alpha} \beta_{B}}\|u\|_{X}$, where $\|B\|$ stands for the norm of the bilinear form $B$. It follows that the direct decomposition $u=u_{0}+u-u_{0}$ into components $u_{0} \in K$ and $u-u_{0} \in K^{\perp_{B}}$ is stable.

As a consequence of Proposition 2.1, there exists a unique continuous projector $P: X \rightarrow X$ with range $K^{\perp_{B}}$ and kernel $K$. We are now going to provide a description of the spectrum of $T$ under the following conditions.

Assumption 2 We assume that

(i) the inclusion $P(X) \hookrightarrow H$ is compact,

(ii) and the inclusion $P(X) \cap T(X) \hookrightarrow X$ is compact.

We notice that, as $P(X)=K^{\perp_{B}}$ is $T$-invariant, the inclusion $T(P(X)) \subset P(X) \cap T(X)$ holds true and Assumption 2 (ii) implies that $T: K^{\perp_{B}} \rightarrow K^{\perp_{B}}$ is compact. The following result is then a consequence of the spectral characterization of compact operators.

Theorem 2.1 Under Assumption 1 and Assumption 2 (ii), the spectrum of $T$ decomposes as follows: $\operatorname{sp}(T)=\{0,1\} \cup\left\{\eta_{k}\right\}_{k \in \mathbb{N}}$, where:

(i) $\eta=1$ is an eigenvalue of $T$ of finite/infinite multiplicity with associated finite/infinite dimensional eigenspace $K$;

(ii) $\left\{\eta_{k}\right\}_{k \in \mathbb{N}} \subset(0,1)$ is a sequence of finite multiplicity eigenvalues of $T$ that converges to 0 and the corresponding eigenspaces lie in $K^{\perp_{B}}$;

iii) if $T$ is non-injective, $\eta=0$ is an eigenvalue of $T$ with associated eigenspace $\operatorname{ker}(T)$.

Remark 2.1 If we assume that $A(v, v) \neq 0$ for all $v \in K^{\perp_{B}} \backslash\{0\}$, then it can also be shown that the ascent of each eigenvalue $\eta_{k} \in(0,1)$ is 1 , c.f. [26, Proposition A.2].

\section{A continuous Galerkin discretization}

We introduce a family $\left\{X_{h}\right\}_{h \geq 0} \subset X$ of finite dimensional subspaces of $X$. The continuous Galerkin discretization of the variational eigenproblem 2.1 reads as follows: find $0 \neq u_{h} \in$ $X_{h}$ and $\kappa_{h} \in \mathbb{R}$ such that

$$
A\left(u_{h}, v\right)=\kappa_{h} B\left(u_{h}, v\right), \quad \forall v \in X_{h} .
$$

We will use the notation

$$
\delta(u, W):=\inf _{w \in W}\|u-w\|_{X},
$$

for the distance in $X$ between an element $u$ and a closed subspace $W \subset X$.

Assumption 3 We assume that $K_{h} \subset K$, where $K_{h}:=\left\{v_{h} \in X_{h} ; \quad c\left(v_{h}, v_{h}\right)=0\right\}$.

We consider $K_{h}^{\perp_{B}}:=\left\{u_{h} \in X_{h}: B\left(u_{h}, v_{h}\right)=0, \forall v_{h} \in K_{h}\right\}$. It is important to notice that $K_{h}^{\perp_{B}}$ is generally not a subspace of $K^{\perp_{B}}$. To proceed with the analysis of problem (3.1) we need the following discrete inf-sup conditions.

Assumption 4 We assume that

(i) there exists $\beta_{A}^{\prime}>0$ independent of $h$ such that $\sup _{v \in X_{h}} \frac{A(u, v)}{\|v\|_{X}} \geq \beta_{A}^{\prime}\|u\|_{X}, \quad \forall u \in X_{h}$, 
(ii) and there exists $\beta_{B}^{\prime}>0$ independent of $h$ such that $\sup _{v \in K_{h}} \frac{B(u, v)}{\|v\|_{H}} \geq \beta_{B}^{\prime}\|u\|_{H}, \quad \forall u \in K_{h}$.

Under Assumption 3 and Assumption 4 (ii), we can prove (as in Proposition 2.1) that the splitting $X_{h}=K_{h} \oplus K_{h}^{\perp_{B}}$ is direct and uniformly stable with respect to $h$. We can also associate to this direct decomposition a unique projector $P_{h}: X_{h} \rightarrow X_{h}$ with range $K_{h}^{\perp_{B}}$ and kernel $K_{h}$, which is uniformly bounded with respect to $h$.

Moreover, thanks to Assumption 4 (i), the linear operator $\tilde{T}_{h}: X \rightarrow X_{h}$ defined, for all $u \in X$, by

$$
A\left(\tilde{T}_{h} u, v\right)=B(u, v), \quad \forall v \in X_{h},
$$

is well-defined and uniformly bounded with respect to $h$. Moreover, we have the Céa estimate (c.f. [18, Lemma 2.28])

$$
\left\|T u-\tilde{T}_{h} u\right\|_{X} \leq\left(1+\frac{\|A\|}{\beta_{A}^{\prime}}\right) \delta\left(T u, X_{h}\right), \quad \forall u \in X .
$$

We point out that $T_{h}:=\left.\tilde{T}_{h}\right|_{X_{h}}$ reduces to the identity on $K_{h}$, which means that 1 is an eigenvalue of $T_{h}$ with associated eigenspace $K_{h}$. Moreover, $\kappa_{h} \neq 0$ is an eigenvalue of Problem (3.1) if and only if $\eta_{h}=1 / \kappa_{h}$ is an eigenvalue of $T_{h}$ and the corresponding eigenspaces are the same. Finally, here again, the symmetry of $T_{h}$ with respect to $B$ implies that $K_{h}^{\perp_{B}}$ is $T_{h}$-invariant, i.e., $T_{h}\left(K_{h}^{\perp_{B}}\right) \subset K_{h}^{\perp_{B}}$. We are then in a position to provide the following spectral decomposition of $T_{h}$.

Theorem 3.1 The spectrum of $T_{h}$ consists of $m:=\operatorname{dim}\left(X_{h}\right)$ eigenvalues, repeated accordingly to their respective multiplicities. Under Assumptions 3 and 4 , it holds $\operatorname{sp}\left(T_{h}\right)=$ $\{1\} \cup\left\{\eta_{h k}\right\}_{k=1}^{m_{0}}$, with $m_{0}=m-\operatorname{dim}\left(K_{h}\right)$. Moreover,

(i) the eigenspace associated to $\eta_{h}=1$ is $K_{h}$;

(ii) $\eta_{h k} \in(0,1), k=1, \ldots, m_{0}-\operatorname{dim}\left(\operatorname{ker}\left(T_{h}\right)\right)$, are eigenvalues with eigenspaces lying in $K_{h}^{\perp_{B}}$;

(iii) if $T_{h}$ is non-injective, $\eta_{h}=0$ is an eigenvalue with corresponding eigenspace $\operatorname{ker}\left(T_{h}\right)$.

Proof The result follows from the decomposition $X_{h}=K_{h} \oplus K_{h}^{\perp}$, the fact that $\left.T_{h}\right|_{K_{h}}$ : $K_{h} \longrightarrow K_{h}$ is the identity and the inclusion $T_{h}\left(K_{h}^{\perp_{B}}\right) \subset K_{h}^{\perp_{B}}$.

Remark 3.1 Here again (see Remark 2.1), if $A(v, v) \neq 0$ for all $v \in K_{h}^{\perp} \backslash\{0\}$ then, the eigenvalues $\eta_{h k} \in(0,1)$ are non-defective.

\section{Correctness of the spectral approximation}

Henceforth, given any positive functions $F_{h}$ and $G_{h}$ depending on the parameter $h$, the abbreviation $F_{h} \lesssim G_{h}$ means that $F_{h} \leq C G_{h}$ with a constant $C>0$ independent $h$. Moreover, the norm of a linear and continuous operator $L: V_{1} \rightarrow V_{2}$ between two Hilbert spaces $V_{1}$ and $V_{2}$ is denoted

$$
\|L\|_{\mathcal{L}\left(V_{1}, V_{2}\right)}:=\sup _{v \in V_{1},\|v\|_{V_{1}}=1}\|L v\|_{V_{2}} .
$$

When $V_{1}=V_{2}=V$ we simply write $\|L\|_{\mathcal{L}(V)}$ for $\|L\|_{\mathcal{L}(V, V)}$. 
The spectral approximation theory developed in [16] for non-compact operators relies essentially on the condition

$$
\lim _{h \rightarrow 0}\left\|T-T_{h}\right\|_{\mathcal{L}\left(X_{h}, X\right)}=0,
$$

to prove that $T_{h}: X_{h} \rightarrow X_{h}$ provides a correct spectral approximation of $T$ (in a sense that will be precised in Theorem 4.1 below). The aim of this section is to show that the following key assumption guarantees (4.1).

Assumption 5 There exists a linear operator $\Xi_{h}: K^{\perp_{B}} \rightarrow X_{h}$ satisfying

(i) there exits a constant $C>0$ independent of $h$ such that

$$
\left\|\Xi_{h} v\right\|_{H} \leq C\|v\|_{H} \quad \text { and } \quad\left\|\Xi_{h} v\right\|_{X} \leq C\|v\|_{X} \quad \forall v \in K^{\perp_{B}}
$$

(ii) $\lim _{h \rightarrow 0}\left\|\left(I-\Xi_{h}\right) P v\right\|_{X}=0, \forall v \in X$,

(iii) and $\left(I-\Xi_{h} P\right) X_{h} \subset K_{h}$.

Lemma 4.1 If Assumptions 1, 3 and 4 are satisfied, the following estimate holds true

$$
\left\|T-T_{h}\right\|_{\mathcal{L}\left(X_{h}, X\right)} \lesssim\left\|P-P_{h}\right\|_{\mathcal{L}\left(X_{h}, X\right)}+\sup _{0 \neq u_{h} \in X_{h}} \frac{\delta\left(T P u_{h}, X_{h}\right)}{\left\|u_{h}\right\|_{X}} .
$$

Proof Taking into account that $T-T_{h}$ vanishes identically on $K_{h} \subset K$ we obtain,

$$
\left(T-T_{h}\right) u_{h}=\left(T-T_{h}\right) P_{h} u_{h}=\left(T-\tilde{T}_{h}\right)\left(P_{h} u_{h}-P u_{h}\right)+\left(T-\tilde{T}_{h}\right) P u_{h}, \quad \forall u_{h} \in X_{h} .
$$

Next, we deduce from the triangle inequality and Céa estimate (3.3) that

$$
\begin{aligned}
\left\|\left(T-T_{h}\right) u_{h}\right\|_{X} & \leq\left(\|T\|_{\mathcal{L}(X)}+\left\|\tilde{T}_{h}\right\|_{\mathcal{L}(X)}\right)\left\|\left(P-P_{h}\right) u_{h}\right\|_{X}+\left\|\left(T-\tilde{T}_{h}\right) P u_{h}\right\|_{X} \\
& \lesssim\left(\|T\|_{\mathcal{L}(X)}+\left\|\tilde{T}_{h}\right\|_{\mathcal{L}(X)}\right)\left\|\left(P-P_{h}\right) u_{h}\right\|_{X}+\delta\left(T P u_{h}, X_{h}\right), \quad \forall u_{h} \in X_{h}
\end{aligned}
$$

and the uniform boundedness of $\widetilde{T}_{h}$ with respect to $h$ gives the result.

To achieve (4.1), let us first prove the following auxiliary result.

Lemma 4.2 Under Assumptions 1 (ii), 3, 4 (ii) and 5 (iii) it holds,

$$
\left\|P-P_{h}\right\|_{\mathcal{L}\left(X_{h}, X\right)} \leq\left(1+\frac{\|B\|}{\beta_{B}^{\prime}}\right)\left\|\left(I-\Xi_{h}\right) P\right\|_{\mathcal{L}\left(X_{h}, H\right)} .
$$

Proof Let us first notice that, by virtue of Assumption 5 (iii),

$$
P_{h} u_{h}-\Xi_{h} P u_{h}=\left(u_{h}-\Xi_{h} P u_{h}\right)-\left(u_{h}-P_{h} u_{h}\right) \in K_{h}, \quad \forall u_{h} \in X_{h} .
$$

The triangle inequality yields

$$
\begin{aligned}
\left\|\left(P-P_{h}\right) u_{h}\right\|_{X} & \leq\left\|P_{h} u_{h}-\Xi_{h} P u_{h}\right\|_{X}+\left\|\left(I-\Xi_{h}\right) P u_{h}\right\|_{X} \\
& \leq \frac{1}{\sqrt{\alpha}}\left(\left\|P P_{h} u_{h}-\Xi_{h} P u_{h}\right\|_{H}+\left\|\left(I-\Xi_{h}\right) P u_{h}\right\|_{H}\right),
\end{aligned}
$$

where the last estimate is a consequence of (4.2), Assumption 5 (iii) and the fact that (I$\left.\Xi_{h}\right) P u_{h}=u_{h}-\Xi_{h} P u_{h}-\left(u_{h}-P u_{h}\right) \in K$. Next, we use the inf-sup condition provided by Assumption 4 (ii) to deduce from (4.3) and Assumption 3 that 


$$
\begin{aligned}
\left\|\left(P-P_{h}\right) u_{h}\right\|_{X} & \leq \frac{1}{\sqrt{\alpha} \beta_{B}^{\prime}} \sup _{v \in K_{h}} \frac{B\left(P_{h} u_{h}-\Xi_{h} P u_{h}, v\right)}{\|v\|_{H}}+\frac{1}{\sqrt{\alpha}}\left\|\left(I-\Xi_{h}\right) P u_{h}\right\|_{H} \\
& =\frac{1}{\sqrt{\alpha} \beta_{B}^{\prime}} \sup _{v \in K_{h}} \frac{B\left(P u_{h}-\Xi_{h} P u_{h}, v\right)}{\|v\|_{H}}+\frac{1}{\sqrt{\alpha}}\left\|\left(I-\Xi_{h}\right) P u_{h}\right\|_{H} \\
& \leq \frac{1}{\sqrt{\alpha}}\left(1+\frac{\|B\|}{\beta_{B}^{\prime}}\right)\left\|\left(I-\Xi_{h}\right) P u_{h}\right\|_{H},
\end{aligned}
$$

and the result follows.

Lemma 4.3 Under Assumptions 1-5 it holds

$$
\lim _{h \rightarrow 0}\left\|T-T_{h}\right\|_{\mathcal{L}\left(X_{h}, X\right)}=0 .
$$

Proof Let us first notice that

$$
\sup _{0 \neq u_{h} \in X_{h}} \frac{\delta\left(T P u_{h}, X_{h}\right)}{\left\|u_{h}\right\|_{X}} \leq \sup _{0 \neq u_{h} \in X_{h}} \frac{\left\|T P u_{h}-\Xi_{h} T P u_{h}\right\|_{X}}{\left\|u_{h}\right\|_{X}} \leq\left\|\left(I-\Xi_{h}\right) T P\right\|_{\mathcal{L}(X)} .
$$

Combining Lemma 4.1 and Lemma 4.2 with the last estimate yields

$$
\left\|T-T_{h}\right\|_{\mathcal{L}\left(X_{h}, X\right)} \lesssim\left\|\left(I-\Xi_{h}\right) T P\right\|_{\mathcal{L}(X)}+\left\|\left(I-\Xi_{h}\right) P\right\|_{\mathcal{L}(X, H)} .
$$

Now, by virtue of Assumption 2 (ii) and Assumption 5 (i)-(ii), $T P: X \rightarrow X$ is compact and the operator $I-\Xi_{h}:\left(K^{\perp_{B}},\|\cdot\|_{X}\right) \rightarrow X$ is uniformly bounded and converges pointwise to zero. Hence, $\left(I-\Xi_{h}\right) T P: X \rightarrow X$ converges uniformly to zero; namely,

$$
\lim _{h \rightarrow 0}\left\|\left(I-\Xi_{h}\right) T P\right\|_{\mathcal{L}(X)}=0 .
$$

On the other hand, thanks to Assumption 2 (i) and Assumption 5 (i)-(ii), $P: X \rightarrow H$ is compact and $I-\Xi_{h}:\left(K^{\perp_{B}},\|\cdot\|_{H}\right) \rightarrow X$ is uniformly bounded and converges pointwise to zero, due to the continuous embedding of $X$ in $H$. Consequently, $\left(I-\Xi_{h}\right) P: X \rightarrow H$ converges uniformly to zero, i.e.,

$$
\lim _{h \rightarrow 0}\left\|\left(I-\Xi_{h}\right) P\right\|_{\mathcal{L}(X, H)}=0,
$$

and the result follows by using (4.5) and (4.6) in (4.4).

For the sake of completeness, we finalize this section by adapting the results of [16] (see also [26]) to show that Assumptions 1-5 are sufficient to ensure the correctness of the spectral approximation. Let us first recall that the resolvent the operator of $T$ is given by

$$
R_{z}(T):=(z I-T)^{-1}: X \longrightarrow X, \quad z \in \mathbb{C} \backslash \operatorname{sp}(T) .
$$

The mapping $z \mapsto\left\|R_{z}(T)\right\|_{\mathcal{L}(X)}$ is continuous for all $z \notin \operatorname{sp}(T)$ and goes to zero as $|z| \rightarrow \infty$. Consequently, it is bounded on any compact subset $F \subset \mathbb{C}$ satisfying $F \cap \operatorname{sp}(T)=\emptyset$, namely, there exists a constant $C_{F}>0$ such that

$$
\left\|R_{z}(T)\right\|_{\mathcal{L}(X)} \leq C_{F}, \quad \forall z \in F .
$$

It is shown in [26, Lemma 1] that the same property holds true uniformly in $h$ for the resolvent $R_{z}\left(T_{h}\right):=\left(z I-T_{h}\right)^{-1}: X_{h} \rightarrow X_{h}$ of the discrete source operator $T_{h}$. We recall this result below. 
Lemma 4.4 Let $F \subset \mathbb{C}$ be an arbitrary compact subset such that $F \cap \operatorname{sp}(T)=\emptyset$. Then, if Assumptions 1-5 are satisfied, there exists $h_{0}>0$ such that $\forall h \leq h_{0}$,

$$
\left\|R_{Z}\left(T_{h}\right)\right\|_{\mathcal{L}\left(X_{h}, X\right)} \leq 2 C_{F}, \quad \forall z \in F,
$$

where $C_{F}>0$ is the constant appearing in (4.8).

Proof We deduce from the decomposition $\left(z I-T_{h}\right) v_{h}=(z I-T) v_{h}+\left(T-T_{h}\right) v_{h}$ and (4.8) that

$$
\left\|\left(z I-T_{h}\right) v_{h}\right\|_{X} \geq\left(C_{F}^{-1}-\left\|T-T_{h}\right\|_{\mathcal{L}\left(X_{h}, X\right)}\right)\left\|v_{h}\right\|_{X}, \quad \forall v_{h} \in X_{h}
$$

and the result follows from Lemma 4.3.

Remark 4.1 Lemma 4.4 means that given an arbitrary compact set $F \subset \mathbb{C} \backslash \operatorname{sp}(T)$ there exists $h_{0}>0$ such that for all $h \leq h_{0}$ it holds $F \subset \mathbb{C} \backslash \operatorname{sp}\left(T_{h}\right)$, which means that, for $h$ small enough, the Galerkin scheme (3.1) does not introduce spurious modes.

For $E$ and $F$ closed subspaces of $X$, we set

$$
\delta(E, F):=\sup _{u \in E:\|u\|_{X}=1} \delta(u, F) \text { and } \widehat{\delta}(E, F):=\max \{\delta(E, F), \delta(F, E)\}
$$

the latter being the so called gap between subspaces $E$ and $F$.

Let $F \subset \mathbb{C} \backslash\{0,1\}$ be a compact set whose boundary $\Lambda$ is a smooth Jordan curve not intersecting $\operatorname{sp}(T)$. It is well known [24] that the linear and bounded operator

$$
\mathcal{E}:=\frac{1}{2 \pi i} \int_{\Lambda} R_{z}(T) d z: X \longrightarrow X
$$

is a projector onto the finite dimensional space $\mathcal{E}(X)$ spanned by the generalized eigenfunctions associated with the finite set of eigenvalues of $T$ contained in $\Lambda$. It follows from Lemma 4.4 that, for $h$ small enough, the linear operator

$$
\mathcal{E}_{h}:=\frac{1}{2 \pi i} \int_{\Lambda} R_{z}\left(T_{h}\right) d z: X_{h} \longrightarrow X_{h}
$$

is uniformly bounded in $h$. Likewise, $\mathcal{E}_{h}$ is a projector onto the $T_{h}$-invariant subspace $\mathcal{E}_{h}\left(X_{h}\right)$ corresponding to the eigenvalues of $T_{h}: X_{h} \rightarrow X_{h}$ contained in $\Lambda$. The aim now is to compare $\mathcal{E}_{h}\left(X_{h}\right)$ to $\mathcal{E}(X)$ in terms of the gap $\widehat{\delta}$. The following auxiliary result is essential for this purpose.

Lemma 4.5 If Assumptions 1-5 are satisfied, there exists $h_{0}>0$ such that

$$
\left\|\mathcal{E}-\mathcal{E}_{h}\right\|_{\mathcal{L}\left(X_{h}, X\right)} \lesssim\left\|T-T_{h}\right\|_{\mathcal{L}\left(X_{h}, X\right)}, \quad \forall h \leq h_{0} .
$$

Proof We reproduce here the proof given in [26, Lemma 2]. By virtue of Lemma 4.4, there exists $h_{0}>0$ such that the resolvent identity

$$
R_{z}(T)-R_{z}\left(T_{h}\right)=R_{z}(T)\left(T-T_{h}\right) R_{z}\left(T_{h}\right), \quad \forall z \in \Lambda, \quad \forall h \leq h_{0},
$$

is satisfied. Hence, for any $v_{h} \in X_{h}$,

$$
\begin{aligned}
\left\|\left(\mathcal{E}-\mathcal{E}_{h}\right) v_{h}\right\|_{X} & \leq \frac{1}{2 \pi} \int_{\Lambda}\left\|\left(R_{z}(T)-R_{z}\left(T_{h}\right)\right) v_{h}\right\|_{X}|d z| \\
& =\frac{1}{2 \pi} \int_{\Lambda}\left\|R_{z}(T)\left(T-T_{h}\right) R_{z}\left(T_{h}\right) v_{h}\right\|_{X}|d z|
\end{aligned}
$$




$$
\leq \frac{1}{2 \pi} \int_{\Lambda}\left\|R_{z}(T)\right\|_{\mathcal{L}(X)}\left\|T-T_{h}\right\|_{\mathcal{L}\left(X_{h}, X\right)}\left\|R_{z}\left(T_{h}\right)\right\|_{\mathcal{L}\left(X_{h}, X\right)}\left\|v_{h}\right\|_{X}|d z|
$$

and the result follows from (4.8) and Lemma 4.4.

Lemma 4.6 Assume that Assumptions 1-5 are satisfied. There exists $h_{0}>0$ such that

$$
\widehat{\delta}\left(\mathcal{E}(X), \mathcal{E}_{h}\left(X_{h}\right)\right) \lesssim\left\|T-T_{h}\right\|_{\mathcal{L}\left(X_{h}, X\right)}+\delta\left(\mathcal{E}(X), X_{h}\right), \quad \forall h \leq h_{0} .
$$

Proof As $\mathcal{E}_{h}: X_{h} \rightarrow X_{h}$ is a projector, it holds $\mathcal{E}_{h} u_{h}=u_{h}$ for all $u_{h} \in \mathcal{E}_{h}\left(X_{h}\right)$. Hence, there exists $h_{0}>0$ such that

$$
\delta\left(u_{h}, \mathcal{E}(X)\right) \leq\left\|\mathcal{E}_{h} u_{h}-\mathcal{E} u_{h}\right\|_{X} \leq\left\|\mathcal{E}_{h}-\mathcal{E}\right\|_{\mathcal{L}\left(X_{h}, X\right)}\left\|u_{h}\right\|_{X}, \quad \forall u_{h} \in \mathcal{E}_{h}\left(X_{h}\right), \quad \forall h \leq h_{0} .
$$

Combining the last estimate with (4.9) gives

$$
\delta\left(\mathcal{E}_{h}\left(X_{h}\right), \mathcal{E}(X)\right) \lesssim\left\|T-T_{h}\right\|_{\mathcal{L}\left(X_{h}, X\right)} .
$$

Using this time that $\mathcal{E} u=u$ for all $u \in \mathcal{E}(X)$ yields

$$
\begin{aligned}
\left\|u-\mathcal{E}_{h} v_{h}\right\|_{X} & \leq\left\|\mathcal{E}\left(u-v_{h}\right)\right\|_{X}+\left\|\left(\mathcal{E}-\mathcal{E}_{h}\right) v_{h}\right\|_{X} \\
& \leq\|\mathcal{E}\|_{\mathcal{L}(X)}\left\|u-v_{h}\right\|_{X}+\left\|\left(\mathcal{E}-\mathcal{E}_{h}\right)\right\|_{\mathcal{L}\left(X_{h}, X\right)}\left\|v_{h}\right\|_{X} \\
& \leq\left(\left\|\mathcal{E}_{h}\right\|_{\mathcal{L}\left(X_{h}, X\right)}+2\|\mathcal{E}\|_{\mathcal{L}(X)}\right)\left\|u-v_{h}\right\|_{X}+\left\|\mathcal{E}-\mathcal{E}_{h}\right\|_{\mathcal{L}\left(X_{h}, X\right)}\|u\|_{X}, \quad \forall v_{h} \in X_{h} .
\end{aligned}
$$

Consequently, by virtue of the uniform boundedness of $\mathcal{E}_{h}: X_{h} \rightarrow X_{h}$, there exists $h_{0}>0$ such that

$$
\delta\left(u, \mathcal{E}_{h}\left(X_{h}\right)\right) \lesssim \delta\left(u, X_{h}\right)+\left\|\mathcal{E}-\mathcal{E}_{h}\right\|_{\mathcal{L}\left(X_{h}, X\right)}, \quad \forall u \in \mathcal{E}(X),\|u\|_{X}=1, \quad \forall h \leq h_{0} .
$$

It follows that

$$
\delta\left(\mathcal{E}(X), \mathcal{E}_{h}\left(X_{h}\right)\right) \lesssim \delta\left(\mathcal{E}(X), X_{h}\right)+\left\|\mathcal{E}-\mathcal{E}_{h}\right\|_{\mathcal{L}\left(X_{h}, X\right)}, \quad \forall h \leq h_{0},
$$

and the result is a consequence of the last estimate, Lemma 4.5 and (4.12).

We are now in a position to establish the convergence properties of the eigenvalues and eigenfunctions.

Theorem 4.1 Assume that Assumptions 1-5 are satisfied. Let $F \subset \mathbb{C} \backslash\{0,1\}$ be an arbitrary compact set with smooth boundary $\Lambda$ satisfying $\Lambda \cap \mathrm{sp}(T)=\emptyset$. We assume that there are $m$ eigenvalues $\eta_{1}^{F}, \ldots, \eta_{m}^{F}$ of $T$ (repeated according to their algebraic multiplicities) contained in $\Lambda$. We also consider the eigenvalues $\eta_{1, h}^{F}, \ldots, \eta_{m(h), h}^{F}$ of $T_{h}: X_{h} \rightarrow X_{h}$ lying in $F$ and repeated according to their algebraic multiplicities. Then, there exists $h_{0}>0$ such that $m(h)=m$ for all $h \leq h_{0}$ and

$$
\lim _{h \rightarrow 0} \max _{1 \leq i \leq m}\left|\eta_{i}^{F}-\eta_{i, h}^{F}\right|=0 .
$$

Moreover, if $\mathcal{E}(X)$ is the $T$-invariant subspace of $X$ spanned by the generalized eigenfunctions corresponding to the set of eigenvalues $\left\{\eta_{i}^{F}, i=1, \ldots, m\right\}$ and $\mathcal{E}_{h}\left(X_{h}\right)$ is the $T_{h}$-invariant subspace of $X_{h}$ spanned by the eigenspaces corresponding to $\left\{\eta_{i, h}, i=1, \ldots, m\right\}$ then $\widehat{\delta}\left(\mathcal{E}(X), \mathcal{E}_{h}\left(X_{h}\right)\right) \rightarrow 0$ as $h \rightarrow 0$.

Proof We deduce from Lemma 4.6, Lemma 4.3 and Assumption 5 (ii) and from the fact that $\mathcal{E}(X) \subset P(X)$ is a finite dimensional subspace of $X$ that

$$
\lim _{h \rightarrow 0} \widehat{\delta}\left(\mathcal{E}(X), \mathcal{E}_{h}\left(X_{h}\right)\right) \lesssim \lim _{h \rightarrow 0}\left\|T-T_{h}\right\|_{\mathcal{L}\left(X_{h}, X\right)}+\lim _{h \rightarrow 0} \delta\left(\mathcal{E}(X), X_{h}\right)=0 .
$$


As a consequence, $\mathcal{E}(X)$ and $\mathcal{E}_{h}\left(X_{h}\right)$ have the same dimension provided $h$ is sufficiently small, c.f. [24]. Finally, as the eigenvalues $\left\{\eta_{1}^{F}, \ldots, \eta_{m}^{F}\right\}$ are isolated, for a sufficiently small $\epsilon>0$, we can consider $D=\cup_{i=1}^{m} D_{\eta_{i}^{F}} \subset F$, where $D_{\eta_{i}^{F}} \subset \mathbb{C}, i=1, \ldots, m$ are disjoint closed disks centered at $\eta_{i}^{F}$ of radius $\epsilon$. The previous analysis shows that there exists $h(\epsilon)>0$ such that $\eta_{1, h}^{F}, \ldots, \eta_{m, h}^{F}$ are all inside of $D$ for $h \leq h(\epsilon)$, which means that

$$
\lim _{h \rightarrow 0} \max _{1 \leq i \leq m}\left|\eta_{i}^{F}-\eta_{i, h}^{F}\right|=0
$$

\section{Asymptotic estimates for the eigenvalue and eigenfunction error}

We proved in Sect. 4 that, under Assumptions 1-5, the Galerkin scheme (3.1) does not pollute the spectrum of $T$ with spurious modes. Moreover, we established the convergence of eigenvalues and eigenfunctions with correct multiplicity. However, in practice the space $\mathcal{E}_{\eta}(X)$ of generalized eigenfunctions corresponding to a given isolated eigenvalue $\eta \neq 1$ enjoys individual smoothness properties. Therefore, in order to be able to claim that the Galerkin method (3.1) has optimal convergence rates we need to estimate the error for a particular eigenvalue $\eta \neq 1$ and for the corresponding eigenspace $\mathcal{E}_{\eta}(X)$ only in terms of $\delta\left(\mathcal{E}_{\eta}(X), X_{h}\right)$. This question has been addressed in [17] for noncompact operators under the condition of coercivity for the bilinear form $A$. In this section we extend the results to the abstract framework we are considering here.

Hereafter, we focus on a particular isolated eigenvalue $\eta \neq 1$ of $T$ of algebraic multiplicity $m$ and let $D_{\eta} \subset \mathbb{C}$ be a closed disk centered at $\eta$ with boundary $\gamma$ such that $D_{\eta} \cap \operatorname{sp}(T)=\{\eta\}$. We denote by $\mathcal{E}_{\eta}:=\frac{1}{2 \pi i} \int_{\gamma} R_{z}(T) d z: X \rightarrow X$ the projector onto the eigenspace $\mathcal{E}_{\eta}(X)$ of $\eta$ and we define, for $h$ small enough, the projector by $\mathcal{E}_{\eta, h}:=\frac{1}{2 \pi i} \int_{\gamma} R_{z}\left(T_{h}\right) d z: X_{h} \rightarrow X_{h}$ onto the $T_{h}$-invariant subspace $\mathcal{E}_{\eta, h}\left(X_{h}\right)$ corresponding to the $m$ eigenvalues of $T_{h}: X_{h} \rightarrow$ $X_{h}$ contained in $\gamma$.

We begin our analysis by proving an analogue of Lemma 4.4 for $R_{z}\left(\tilde{T}_{h}\right):=\left(z I-\tilde{T}_{h}\right)^{-1}$ : $X \longrightarrow X$.

Lemma 5.1 Assume that Assumptions 1-5 are satisfied. Let $F \subset \mathbb{C}$ be an arbitrary compact subset such that $F \cap \operatorname{sp}(T)=\emptyset$. There exist $C_{F}^{\prime}>0$ and $h_{0}>0$ such that,

$$
\left\|R_{z}\left(\tilde{T}_{h}\right)\right\|_{\mathcal{L}(X)} \lesssim C_{F}^{\prime}, \quad \forall z \in F, \quad \forall h \leq h_{0}
$$

Proof Given $u \in X$ we let $u_{h}^{*}=\tilde{T}_{h} u \in X_{h}$. We deduce from the identity

$$
\left(z I-T_{h}\right) u_{h}^{*}=\tilde{T}_{h}\left(z I-\tilde{T}_{h}\right) u
$$

and from Lemma 4.4 that

$$
\left\|u_{h}^{*}\right\|_{X} \leq 2 C_{F}\left\|\left(z I-T_{h}\right) u_{h}^{*}\right\|_{X} \leq 2 C_{F}\left\|\tilde{T}_{h}\right\|_{\mathcal{L}(X)}\left\|\left(z I-\tilde{T}_{h}\right) u\right\|_{X}, \quad \forall h \leq h_{0} .
$$

The last estimate and the triangle inequality yield

$$
|z|\|u\|_{X} \leq\left\|u_{h}^{*}\right\|_{X}+\left\|\left(z I-\tilde{T}_{h}\right) u\right\|_{X} \leq\left(1+2 C_{F}\left\|\tilde{T}_{h}\right\|_{\mathcal{L}(X)}\right)\left\|\left(z I-\tilde{T}_{h}\right) u\right\|_{X}, \quad \forall u \in X
$$

and the result follows from the uniform boundedness of $\left\|\tilde{T}_{h}\right\|_{\mathcal{L}(X)}$. 
It follows from Lemma 5.1 that, for $h$ small enough, $R_{z}\left(\tilde{T}_{h}\right): X \longrightarrow X$ is linear and bounded uniformly in $h$ for all $z \in \gamma$. Hence, the linear operator

$$
\tilde{\mathcal{E}}_{\eta, h}:=\frac{1}{2 \pi i} \int_{\gamma} R_{z}\left(\tilde{T}_{h}\right) d z: X \longrightarrow X
$$

is uniformly bounded as well. It is straightforward that $\left.R_{z}\left(\tilde{T}_{h}\right)\right|_{X_{h}}=R_{z}\left(T_{h}\right)$. It follows that we also have $\left.\tilde{\mathcal{E}}_{\eta, h}\right|_{X_{h}}=\mathcal{E}_{\eta, h}$. Moreover, if $\tilde{\eta} \in D_{\eta}$ is an eigenvalue of $\tilde{T}_{h}$, as $\tilde{\eta} \neq 0$, the corresponding eigenspace is a subspace of $\mathcal{E}_{\eta, h}\left(X_{h}\right)$ and $\tilde{\eta}$ should necessarily coincide with one of the eigenvalues $\left\{\eta_{i, h}, i=1, \ldots, m\right\}$ of $T_{h}$. We conclude that $\tilde{\mathcal{E}}_{\eta, h}(X)=\mathcal{E}_{\eta, h}\left(X_{h}\right)$ is the eigenspace corresponding to the eigenvalues of $T_{h}$ contained in $\gamma$.

Theorem 5.1 Under Assumptions 1-5 and for $h$ small enough, it holds

$$
\widehat{\delta}\left(\mathcal{E}_{\eta}(X), \mathcal{E}_{\eta, h}\left(X_{h}\right)\right) \lesssim \delta\left(\mathcal{E}_{\eta}(X), X_{h}\right)
$$

Proof Thanks to Lemma 5.1, there exists $h_{0}>0$ such that

$$
R_{z}(T)-R_{z}\left(\tilde{T}_{h}\right)=R_{z}\left(\tilde{T}_{h}\right)\left(T-\tilde{T}_{h}\right) R_{z}(T), \quad \forall z \in \gamma, \quad \forall h \leq h_{0} .
$$

Hence, recalling that $\mathcal{E}_{\eta}(X)$ is invariant for $T$ and hence also for $R_{z}(T)$ (i.e. $R_{z}(T) \mathcal{E}_{\eta}(X) \subset$ $\left.\mathcal{E}_{\eta}(X)\right)$, we have

$$
\begin{aligned}
\left\|\mathcal{E}_{\eta}-\tilde{\mathcal{E}}_{\eta, h}\right\|_{\mathcal{L}\left(\mathcal{E}_{\eta}(X), X\right)} & \leq \frac{1}{2 \pi} \int_{\gamma}\left\|R_{z}(T)-R_{z}\left(\tilde{T}_{h}\right)\right\|_{\mathcal{L}\left(\mathcal{E}_{\eta}(X), X\right)}|d z| \\
& =\frac{1}{2 \pi} \int_{\gamma}\left\|R_{z}\left(\tilde{T}_{h}\right)\left(T-\tilde{T}_{h}\right) R_{z}(T)\right\|_{\mathcal{L}\left(\mathcal{E}_{\eta}(X), X\right)}|d z| \\
& \leq \frac{1}{2 \pi} \int_{\gamma}\left\|R_{z}\left(\tilde{T}_{h}\right)\right\|_{\mathcal{L}(X)}\left\|T-\tilde{T}_{h}\right\|_{\mathcal{L}\left(\mathcal{E}_{\eta}(X), X\right)}\left\|R_{z}(T)\right\|_{\mathcal{L}\left(\mathcal{E}_{\eta}(X), X\right)}|d z| \\
& \lesssim\left\|\left(T-\tilde{T}_{h}\right)\right\|_{\mathcal{L}\left(\mathcal{E}_{\eta}(X), X\right) .}
\end{aligned}
$$

Now, due to the fact that $\mathcal{E}_{\eta}(X) \subset K^{\perp_{B}}$ is finite dimensional and $T$-invariant, we deduce from (5.2), Céa estimate (3.3) and Assumption 5 (ii) that

$$
\left\|\mathcal{E}_{\eta}-\tilde{\mathcal{E}}_{\eta, h}\right\|_{\mathcal{L}\left(\mathcal{E}_{\eta}(X), X\right)} \lesssim \delta\left(\mathcal{E}_{\eta}(X), X_{h}\right) \rightarrow 0, \quad \text { as } h \rightarrow 0 .
$$

It follows that

$$
\delta\left(\mathcal{E}_{\eta}(X), \mathcal{E}_{\eta, h}\left(X_{h}\right)\right)=\delta\left(\mathcal{E}_{\eta}(X), \tilde{\mathcal{E}}_{\eta, h}(X)\right) \leq\left\|\mathcal{E}_{\eta}-\tilde{\mathcal{E}}_{\eta, h}\right\|_{\mathcal{L}\left(\mathcal{E}_{\eta}(X), X\right)} \lesssim \delta\left(\mathcal{E}_{\eta}(X), X_{h}\right) .
$$

On the other hand, we also deduce from (5.3) that the operator $\tilde{\mathcal{E}}_{\eta, h}: \mathcal{E}_{\eta}(X) \rightarrow \mathcal{E}_{\eta, h}\left(X_{h}\right)$ converges uniformly to the identity, which proves that it is invertible, for $h$ small enough. We denote its inverse $\Lambda_{\eta, h}: \mathcal{E}_{\eta, h}\left(X_{h}\right) \rightarrow \mathcal{E}_{\eta}(X)$. It is straightforward that, if $h_{0}>0$ is such that

$$
\left\|I-\tilde{\mathcal{E}}_{\eta, h}\right\|_{\mathcal{L}\left(\mathcal{E}_{\eta}(X), X\right)} \leq 1 / 2, \quad\left(\forall h \leq h_{0}\right)
$$

then $\left\|\Lambda_{\eta, h}\right\|_{\mathcal{L}\left(\mathcal{E}_{\eta, h}\left(X_{h}\right), X\right)} \leq 2$ and, again by (5.3),

$$
\begin{aligned}
\delta\left(\mathcal{E}_{\eta, h}\left(X_{h}\right), \mathcal{E}_{\eta}(X)\right) & =\delta\left(\tilde{\mathcal{E}}_{\eta, h}(X), \mathcal{E}_{\eta}(X)\right) \leq\left\|I-\Lambda_{h}\right\|_{\mathcal{L}\left(\mathcal{E}_{\eta, h}\left(X_{h}\right), X\right)} \\
& =\left\|\left(\tilde{\mathcal{E}}_{\eta, h}-I\right) \Lambda_{h}\right\|_{\mathcal{L}\left(\mathcal{E}_{\eta, h}\left(X_{h}\right), X\right)}
\end{aligned}
$$




$$
\leq 2\left\|\tilde{\mathcal{E}}_{\eta, h}-I\right\|_{\mathcal{L}\left(\mathcal{E}_{\eta}(X), X\right)}=2\left\|\tilde{\mathcal{E}}_{\eta, h}-\mathcal{E}_{\eta}\right\|_{\mathcal{L}\left(\mathcal{E}_{\eta}(X), X\right)} \lesssim \delta\left(\mathcal{E}_{\eta}(X), X_{h}\right) .
$$

The result follows from the last estimate and (5.4).

We recall that $\kappa=1 / \eta$ an eigenvalue of Problem 2.1 with the same m-dimensional eigenspace $\mathcal{E}_{\eta}(X)$. Analogously, if $\eta_{i, h}, i=1, \ldots, m$, are the eigenvalues of $T_{h}$ (repeated accordingly to their respective algebraic multiplicities) that converge to $\eta$ then, $\kappa_{i, h}=1 / \eta_{i, h}$ are the eigenvalues of Problem 3.1 converging to $\kappa$ and the corresponding generalized eigenfunctions span $\mathcal{E}_{\eta, h}\left(X_{h}\right)$. The last step of this section is the following theorem, in which we establish a double order of convergence for the eigenvalues. To this end we need the following assumption.

Assumption 6 Assume $B(u, u)>0$ for all $u \in \mathcal{E}_{\eta}(X) \backslash\{0\}$.

Theorem 5.2 Under Assumptions 1-6, there exists $h_{0}>0$ such that,

$$
\max _{1 \leq i \leq m}\left|\kappa_{i, h}-\kappa\right| \lesssim \delta\left(\mathcal{E}_{\eta}(X), X_{h}\right)^{2}, \quad \forall h \leq h_{0}
$$

Proof We denote by $u_{i, h}$ an eigenfunction corresponding to $\kappa_{i, h}$ satisfying $\left\|u_{i, h}\right\|_{X}=1$. There exists an eigenfunction $u \in \mathcal{E}_{\eta}(X)$ satisfying

$$
\left\|u_{i, h}-u\right\|_{X}=\delta\left(u_{i, h}, \mathcal{E}_{\eta}(X)\right) \leq \widehat{\delta}\left(\mathcal{E}_{\eta, h}\left(X_{h}\right), \mathcal{E}_{\eta}(X)\right) \lesssim \delta\left(\mathcal{E}_{\eta}(X), X_{h}\right) \rightarrow 0 \text { as } h \rightarrow 0 .
$$

It follows that, for $h$ small enough, $\|u\|_{X}$ is bounded from below and above by a constant independent of $h$. Furthermore, Assumption 6 and the fact that $\mathcal{E}_{\eta}(X)$ is finite-dimensional imply the existence of $c>0$, independent of $h$, such that $B(u, u) \geq c\|u\|_{X}$ for all $u \in$ $\mathcal{E}_{\eta}(X)$. Using (5.5) and the uniform boundedness of $\|u\|_{X}$, it is straightforward deduce that $B\left(u_{i h}, u_{i h}\right) \geq \frac{c}{2}$ for $h$ sufficiently small. We can now use the identity

$$
A\left(u-u_{i, h}, u-u_{i, h}\right)-\kappa B\left(u-u_{i, h}, u-u_{i, h}\right)=\left(\kappa_{i, h}-\kappa\right) B\left(u_{i, h}, u_{i, h}\right)
$$

to obtain the estimate

$$
\begin{aligned}
\frac{c}{2}\left|\kappa_{i, h}-\kappa\right| & \leq\left|A_{h}\left(u-u_{i, h}, u-u_{i, h}\right)\right|+|\kappa|\left|B\left(u-u_{i, h}, u-u_{i, h}\right)\right| \\
& \lesssim\left\|u-u_{i, h}\right\|_{X}^{2} \lesssim \delta\left(\mathcal{E}_{\eta}(X), X_{h}\right)^{2}, \quad \forall i=1, \ldots, m
\end{aligned}
$$

and the result follows.

\section{Applications}

We present two applications of the abstract theory developed in the previous sections. They concern dual mixed formulations for the elasticity and Stokes eigensystems.

We denote the space of real matrices of order $d \times d$ by $\mathbb{M}$, and define $\mathbb{S}:=\{\tau \in$ $\left.\mathbb{M} ; \boldsymbol{\tau}=\boldsymbol{\tau}^{\mathrm{t}}\right\}$ and $\mathbb{K}:=\left\{\boldsymbol{\tau} \in \mathbb{M} ; \boldsymbol{\tau}=-\boldsymbol{\tau}^{\mathrm{t}}\right\}$ as the subspaces of real symmetric and skew symmetric matrices, respectively. The component-wise inner product of two matrices $\boldsymbol{\sigma}, \boldsymbol{\tau} \in \mathbb{M}$ is defined by $\boldsymbol{\sigma}: \boldsymbol{\tau}:=\operatorname{tr}\left(\boldsymbol{\sigma}^{\mathrm{t}} \boldsymbol{\tau}\right)$, where $\operatorname{tr} \boldsymbol{\tau}:=\sum_{i=1}^{d} \tau_{i i}$ and $\boldsymbol{\tau}^{\mathrm{t}}:=\left(\tau_{j i}\right)$ stand for the trace and the transpose of $\tau=\left(\tau_{i j}\right)$, respectively. We also introduce the deviatoric part $\boldsymbol{\tau}^{\mathrm{D}}:=\boldsymbol{\tau}-\frac{1}{d}(\operatorname{tr} \boldsymbol{\tau}) I$ of a tensor $\boldsymbol{\tau}$, where $I$ stands here for the identity in $\mathbb{M}$. 
Along this paper we convene to apply all differential operators row-wise. Hence, given regular tensors $\sigma: \Omega \rightarrow \mathbb{M}$ and vector fields $\boldsymbol{u}: \Omega \rightarrow \mathbb{R}^{d}$, we set the divergence $\operatorname{div} \sigma$ : $\Omega \rightarrow \mathbb{R}^{d}$, the gradient $\nabla \boldsymbol{u}: \Omega \rightarrow \mathbb{M}$, and the linearized strain tensor $\boldsymbol{\varepsilon}(\boldsymbol{u}): \Omega \rightarrow \mathbb{S}$ as

$$
(\operatorname{div} \boldsymbol{\sigma})_{i}:=\sum_{j} \partial_{j} \sigma_{i j}, \quad(\nabla \boldsymbol{u})_{i j}:=\partial_{j} u_{i}, \quad \text { and } \boldsymbol{\varepsilon}(\boldsymbol{u}):=\frac{1}{2}\left[\nabla \boldsymbol{u}+(\nabla \boldsymbol{u})^{\mathrm{t}}\right] .
$$

Let $D$ be a polyhedral Lipschitz bounded domain of $\mathbb{R}^{d}(d=2,3)$, with boundary $\partial D$. For $s \in \mathbb{R}, H^{s}(D, E)$ stands for the usual Hilbertian Sobolev space of functions with domain $D$ and values in $\mathrm{E}$, where $E$ is either $\mathbb{R}, \mathbb{R}^{d}$ or $\mathbb{M}$. In the case $E=\mathbb{R}$ we simply write $H^{s}(D)$. The norm of $H^{s}(D, E)$ is denoted $\|\cdot\|_{s, D}$ indistinctly for $E=\mathbb{R}, \mathbb{R}^{d}, \mathbb{M}$. We use the convention $H^{0}(D, E):=L^{2}(D, E)$ and let $(\cdot, \cdot)_{D}$ be the inner product in $L^{2}(D, E)$, for $E=\mathbb{R}, \mathbb{R}^{d}, \mathbb{M}$, namely,

$$
(\boldsymbol{u}, \boldsymbol{v})_{D}:=\int_{D} \boldsymbol{u} \cdot \boldsymbol{v}, \forall \boldsymbol{u}, \boldsymbol{v} \in L^{2}\left(D, \mathbb{R}^{d}\right), \quad(\boldsymbol{\sigma}, \boldsymbol{\tau})_{D}:=\int_{D} \boldsymbol{\sigma}: \boldsymbol{\tau}, \forall \boldsymbol{\sigma}, \boldsymbol{\tau} \in L^{2}(D, \mathbb{M}) .
$$

We denote by $H(\operatorname{div}, D, \mathbb{M})$ the space of functions in $L^{2}(D, \mathbb{M})$ with divergence in $L^{2}\left(D, \mathbb{R}^{d}\right)$. We equip this Hilbert space with the norm $\|\boldsymbol{\tau}\|_{\mathbf{d i v}, D}^{2}:=\|\boldsymbol{\tau}\|_{0, D}^{2}+\|\operatorname{div} \boldsymbol{\tau}\|_{0, D}^{2}$. Finally, $H\left(\mathbf{d i v}^{0}, D, \mathbb{M}\right)$ stands for the subspace of divergence free tensors in $H(\operatorname{div}, D, \mathbb{M})$, i.e.,

$$
H\left(\operatorname{div}^{0}, D, \mathbb{M}\right):=\{\tau \in H(\operatorname{div}, D, \mathbb{M}), \operatorname{div} \tau=0 \text { in } D\}
$$

\subsection{Stress formulation of the elasticity eigenproblem with reduced symmetry}

\subsubsection{The continuous problem}

Let $\Omega \subset \mathbb{R}^{d}(d=2,3)$ be a bounded Lipschitz polygon/polyhedron representing a linearly elastic body with mass density $\varrho \in L^{\infty}(\Omega)$ satisfying $\varrho(x) \geq \varrho_{0}>0$ a.e. in $\Omega$. For simplicity, we assume that the structure is fixed at the boundary $\partial \Omega$. We denote by $\mathcal{A}(\boldsymbol{x})$ : $\mathbb{M} \rightarrow \mathbb{M}$ the symmetric and positive-definite 4th-order tensor (known as the compliance tensor) that relates the Cauchy stress tensor $\sigma$ to the strain tensor through the linear material law $\mathcal{A}(\boldsymbol{x}) \boldsymbol{\sigma}=\boldsymbol{\varepsilon}(\boldsymbol{u})$.

Our aim is to find natural frequencies $\omega \in \mathbb{R}$ such that $\operatorname{div} \boldsymbol{\sigma}+\omega^{2} \varrho(\boldsymbol{x}) \boldsymbol{u}=0$ in $\Omega$. Here, we opt for combining this equilibrium equation with the constitutive law to eliminate the displacement field $\boldsymbol{u}$ and impose $\sigma$ as a primary variable. This procedure leads to the following grad-div eigensystem: Find $0 \neq \sigma: \Omega \rightarrow \mathbb{S}, 0 \neq \boldsymbol{r}: \Omega \rightarrow \mathbb{K}$ and eigenmodes $\omega \in \mathbb{R}$ such that,

$$
\begin{aligned}
-\nabla\left(\varrho^{-1} \operatorname{div} \sigma\right) & =\omega^{2}(\mathcal{A} \boldsymbol{\sigma}+\boldsymbol{r}) \quad \text { in } \Omega, \\
\varrho^{-1} \operatorname{div} \sigma & =0 \quad \text { on } \partial \Omega .
\end{aligned}
$$

We notice that we introduced above the skew symmetric tensor $\boldsymbol{r}:=\frac{1}{2}\left[\nabla \boldsymbol{u}-(\nabla \boldsymbol{u})^{\mathrm{t}}\right]$ (the rotation) by writing Hooke's law $\mathcal{A} \boldsymbol{\sigma}=\nabla \boldsymbol{u}-\boldsymbol{r}$. This additional unknown will act as a Lagrange multiplier for the symmetry restriction.

The variational formulation of the spectral problem (6.1) can be cast into the abstract framework presented in Sect. 2 by defining problem (2.1) with $\kappa=1+\omega^{2}, H:=L^{2}(\Omega, \mathbb{M}) \times$ $L^{2}(\Omega, \mathbb{M}), X:=H(\operatorname{div}, \Omega, \mathbb{M}) \times L^{2}(\Omega, \mathbb{K})$ and with bounded and symmetric bilinear forms 
$B: H \times H \rightarrow \mathbb{R}, c: X \times X \rightarrow \mathbb{R}$ and $A: X \times X \rightarrow \mathbb{R}$ given by

$$
\begin{aligned}
B((\boldsymbol{\sigma}, \boldsymbol{r}),(\boldsymbol{\tau}, \boldsymbol{s})) & :=(\mathcal{A} \boldsymbol{\sigma}, \boldsymbol{\tau})_{\Omega}+(\boldsymbol{r}, \boldsymbol{\tau})_{\Omega}+(\boldsymbol{s}, \boldsymbol{\sigma})_{\Omega}, \quad c((\boldsymbol{\sigma}, \boldsymbol{r}),(\boldsymbol{\tau}, \boldsymbol{s})) \\
& :=\left(\varrho^{-1} \operatorname{div} \boldsymbol{\sigma}, \operatorname{div} \boldsymbol{\tau}\right)_{\Omega} \\
A((\boldsymbol{\sigma}, \boldsymbol{r}),(\boldsymbol{\tau}, \boldsymbol{s})) & :=c((\boldsymbol{\sigma}, \boldsymbol{r}),(\boldsymbol{\tau}, \boldsymbol{s}))+B((\boldsymbol{\sigma}, \boldsymbol{r}),(\boldsymbol{\tau}, \boldsymbol{s})),
\end{aligned}
$$

c.f. [26] for more details. The Hilbert spaces $H$ and $X$ are endowed with the norms

$$
\|(\boldsymbol{\tau}, \boldsymbol{s})\|_{H}^{2}:=\|\boldsymbol{\tau}\|_{0, \Omega}^{2}+\|\boldsymbol{s}\|_{0, \Omega}^{2}, \quad\|(\boldsymbol{\tau}, \boldsymbol{s})\|_{X}^{2}:=\|\boldsymbol{\tau}\|_{\operatorname{div}, \Omega}^{2}+\|\boldsymbol{s}\|_{0, \Omega}^{2} .
$$

We point out that the continuous inclusion $X \hookrightarrow H$ is not compact.

We proceed now to check out Assumption 1 and Assumption 2. We begin by noticing that

$$
K:=\{(\boldsymbol{\tau}, \boldsymbol{s}) \in X, c((\boldsymbol{\tau}, \boldsymbol{s}),(\boldsymbol{\tau}, \boldsymbol{s}))=0\}=H\left(\operatorname{div}^{0}, \Omega, \mathbb{M}\right) \times L^{2}(\Omega, \mathbb{K})
$$

is not a finite dimensional subspace of $X$. It is well-known that the bilinear form $(\boldsymbol{\tau},(\boldsymbol{v}, \boldsymbol{s})) \mapsto$ $(\operatorname{div} \boldsymbol{\tau}, \boldsymbol{u})_{\Omega}+(\boldsymbol{\tau}, \boldsymbol{s})_{\Omega}$ satisfies the inf-sup condition for the pair $\left\{H(\operatorname{div}, \Omega, \mathbb{M}), L^{2}\left(\Omega, \mathbb{R}^{d}\right) \times\right.$ $\left.L^{2}(\Omega, \mathbb{K})\right\}$, which can be equivalently formulated as follows, (c.f. [10, Proposition 2] ).

Lemma 6.1 There exists a linear and bounded operator $\Theta: L^{2}\left(\Omega, \mathbb{R}^{d}\right) \times L^{2}(\Omega, \mathbb{K}) \rightarrow$ $H(\operatorname{div}, \Omega, \mathbb{M})$ such that

$$
(\operatorname{div} \Theta(\boldsymbol{v}, \boldsymbol{s}), \boldsymbol{v})_{\Omega}+(\Theta(\boldsymbol{v}, \boldsymbol{s}), \boldsymbol{s})_{\Omega}=\|\boldsymbol{v}\|_{0, \Omega}^{2}+\|\boldsymbol{s}\|_{0, \Omega}^{2} \quad \forall(\boldsymbol{v}, \boldsymbol{s}) \in L^{2}\left(\Omega, \mathbb{R}^{d}\right) \times L^{2}(\Omega, \mathbb{K}) .
$$

Corollary 6.1 Assumption 1 is satisfied.

Proof It follows from Lemma 6.1 that

$$
\begin{aligned}
\sup _{\boldsymbol{\tau} \in H(\operatorname{div}, \Omega, \mathbb{M})} \frac{(\boldsymbol{\tau}, \boldsymbol{s})_{\Omega}}{\|\boldsymbol{\tau}\|_{\operatorname{div}, \Omega}} & \geq \sup _{\boldsymbol{\tau} \in H\left(\operatorname{div}^{0}, \Omega, \mathbb{M}\right)} \frac{(\boldsymbol{\tau}, \boldsymbol{s})_{\Omega}}{\|\boldsymbol{\tau}\|_{0, \Omega}} \geq \frac{(\Theta(0, s), s)_{\Omega}}{\|\Theta(0, s)\|_{0, \Omega}}=\frac{\|\boldsymbol{s}\|_{0, \Omega}^{2}}{\|\Theta(0, s)\|_{0, \Omega}} \\
& \geq \frac{1}{\|\Theta\|}\|\boldsymbol{s}\|_{0, \Omega},
\end{aligned}
$$

for all $s \in L^{2}(\Omega, \mathbb{K})$. This means that the bilinear form $(\boldsymbol{\tau}, \boldsymbol{s})_{\Omega}$ satisfies the inf-sup condition for the pair $\left\{H\left(\operatorname{div}^{0}, \Omega, \mathbb{M}\right), L^{2}(\Omega, \mathbb{K})\right\}$ and also for the pair $\left\{H(\operatorname{div}, \Omega, \mathbb{M}), L^{2}(\Omega, \mathbb{K})\right\}$. Moreover, we have that $(\boldsymbol{\sigma}, \boldsymbol{\tau}) \mapsto(\mathcal{A} \boldsymbol{\sigma}, \boldsymbol{\tau})_{\Omega}$ is coercive on $H\left(\operatorname{div}^{0}, \Omega, \mathbb{M}\right)$ while $(\boldsymbol{\sigma}, \boldsymbol{\tau}) \mapsto$ $(\mathcal{A} \boldsymbol{\sigma}, \boldsymbol{\tau})+\left(\varrho^{-1} \boldsymbol{d i v} \boldsymbol{\sigma}, \boldsymbol{d i v} \boldsymbol{\tau}\right)_{\Omega}$ is coercive on the whole space $H(\operatorname{div}, \Omega, \mathbb{M})$. By virtue of the Babuška-Brezzi theory [11], for all $L \in X^{\prime}$, the saddle point problem: find $(\sigma, r) \in X$ such that

$$
A((\boldsymbol{\sigma}, \boldsymbol{r}),(\boldsymbol{\tau}, \boldsymbol{s}))=L((\boldsymbol{\tau}, \boldsymbol{s})), \quad \forall(\boldsymbol{\tau}, \boldsymbol{s}) \in X,
$$

is well-posed, which implies that Assumption 1 (i) is satisfied. Likewise, for all $L_{0} \in K^{\prime}$, the saddle point problem: find $(\sigma, \boldsymbol{r}) \in K=H\left(\operatorname{div}^{0}, \Omega, \mathbb{M}\right) \times L^{2}(\Omega, \mathbb{K})$ such that

$$
B((\boldsymbol{\sigma}, \boldsymbol{r}),(\boldsymbol{\tau}, \boldsymbol{s}))=L_{0}((\boldsymbol{\tau}, \boldsymbol{s})), \quad \forall(\boldsymbol{\tau}, \boldsymbol{s}) \in K,
$$

is well-posed. Consequently, Assumption 1 (ii) also holds true.

Thanks to Corollary 6.1, we can define the source operator $T: X \rightarrow X$ in terms of problem (2.2). We recall that $\operatorname{ker}(I-T)=K$ and that the symmetry of $T$ with respect to $B(\cdot, \cdot)$ yields $T\left(K^{\perp_{B}}\right) \subset K^{\perp_{B}}$. In addition, the direct and stable splitting $X=K \oplus K^{\perp_{B}}$ holds true. Our aim now is to characterize the unique projector $P: X \rightarrow X$ with range $K^{\perp_{B}}$ and kernel $K$ associated to this splitting. 
For any $(\boldsymbol{\sigma}, \boldsymbol{r}) \in X$, we consider $P(\boldsymbol{\sigma}, \boldsymbol{r}):=(\widetilde{\boldsymbol{\sigma}}, \widetilde{\boldsymbol{r}})$ with $\tilde{\boldsymbol{\sigma}}=\mathcal{A}^{-1} \boldsymbol{\varepsilon}(\widetilde{\boldsymbol{u}})$ and $\widetilde{\boldsymbol{r}}:=\frac{1}{2}\left[\nabla \widetilde{\boldsymbol{u}}-(\nabla \tilde{\boldsymbol{u}})^{\mathrm{t}}\right]$, where $\tilde{\boldsymbol{u}}$ is the unique solution of the classical displacement based variational formulation of the elasticity problem in $\Omega$ with volume load $\operatorname{div} \sigma$, namely, $\tilde{\boldsymbol{u}} \in H_{0}^{1}\left(\Omega, \mathbb{R}^{d}\right)$ solves

$$
\left(\mathcal{A}^{-1} \boldsymbol{\varepsilon}(\widetilde{\boldsymbol{u}}), \boldsymbol{\varepsilon}(\boldsymbol{v})\right)_{\Omega}=(\operatorname{div} \boldsymbol{\sigma}, \boldsymbol{v})_{\Omega}, \quad \forall \boldsymbol{v} \in H_{0}^{1}\left(\Omega, \mathbb{R}^{d}\right) .
$$

We point out that $\operatorname{div} \widetilde{\sigma}=\operatorname{div} \sigma$ by construction. In addition, Korn's inequality provides the stability estimate

$$
\|\tilde{u}\|_{1, \Omega} \leq C\|\operatorname{div} \boldsymbol{\sigma}\|_{0, \Omega},
$$

which ensures the continuity of $P: X \rightarrow X$. Now, it is clear that $P \circ P=P$ and ker $P=K$. Besides, for any $(\boldsymbol{\sigma}, \boldsymbol{r}) \in X$,

$$
B(P(\boldsymbol{\sigma}, \boldsymbol{r}),(\boldsymbol{\tau}, \boldsymbol{s}))=B((\widetilde{\boldsymbol{\sigma}}, \widetilde{\boldsymbol{r}}),(\boldsymbol{\tau}, \boldsymbol{s}))=(\nabla \widetilde{\boldsymbol{u}}, \boldsymbol{\tau})_{\Omega}=0, \quad \forall(\boldsymbol{\tau}, \boldsymbol{s}) \in K,
$$

which proves that $P(X) \subset K^{\perp_{B}}$. Finally, we notice that $(I-P) X \subset K$, and hence, $K^{\perp_{B}}=P\left(K^{\perp_{B}}\right)+(I-P) K^{\perp_{B}}=P\left(K^{\perp_{B}}\right) \subset P(X)$. We conclude that $P: X \rightarrow X$ is indeed the unique continuous projector corresponding to the direct and stable decomposition $X=K \oplus K^{\perp_{B}}$.

\section{Lemma 6.2 Assumption 2 is satisfied.}

Proof Let $\left\{\left(\sigma_{n}, \boldsymbol{r}_{n}\right)\right\}_{n}$ be a weakly convergent sequence in $X$. As $P \in \mathcal{L}(X)$, the sequence $\left\{\left(\widetilde{\boldsymbol{\sigma}}_{n}, \widetilde{\boldsymbol{r}}_{n}\right)\right\}_{n}:=\left\{P\left(\left(\boldsymbol{\sigma}_{n}, \boldsymbol{r}_{n}\right)\right)\right\}_{n}$ is also weakly convergent in $X$. By definition, $\widetilde{\boldsymbol{\sigma}}_{n}=$ $\mathcal{A}^{-1} \boldsymbol{\varepsilon}\left(\widetilde{\boldsymbol{u}}_{n}\right)$ and $\widetilde{\boldsymbol{r}}_{n}:=\frac{1}{2}\left[\nabla \widetilde{\boldsymbol{u}}_{n}-\left(\nabla \widetilde{\boldsymbol{u}}_{n}\right)^{\mathrm{t}}\right]$, where $\widetilde{\boldsymbol{u}}_{n} \in H_{0}^{1}\left(\Omega, \mathbb{R}^{d}\right)$ solves (6.4) with righthand side $\operatorname{div} \boldsymbol{\sigma}_{n}$. It follows from (6.5) that $\widetilde{\boldsymbol{u}}_{n}$ is bounded in $H_{0}^{1}\left(\Omega, \mathbb{R}^{d}\right)$ and the compactness of the embedding $H^{1}\left(\Omega, \mathbb{R}^{d}\right) \hookrightarrow L^{2}\left(\Omega, \mathbb{R}^{d}\right)$ implies that $\left\{\tilde{\boldsymbol{u}}_{n}\right\}_{n}$ admits a subsequence (denoted again $\left\{\widetilde{\boldsymbol{u}}_{n}\right\}_{n}$ ) that converges strongly in $L^{2}\left(\Omega, \mathbb{R}^{d}\right)$. Next, we deduce from the Green identity

$$
\left(\mathcal{A}\left(\widetilde{\boldsymbol{\sigma}}_{p}-\tilde{\boldsymbol{\sigma}}_{q}\right), \tilde{\boldsymbol{\sigma}}_{p}-\tilde{\boldsymbol{\sigma}}_{q}\right)_{\Omega}=\left(\boldsymbol{\varepsilon}\left(\tilde{\boldsymbol{u}}_{p}-\tilde{\boldsymbol{u}}_{q}\right), \tilde{\boldsymbol{\sigma}}_{p}-\tilde{\boldsymbol{\sigma}}_{q}\right)_{\Omega}=-\left(\widetilde{\boldsymbol{u}}_{p}-\tilde{\boldsymbol{u}}_{q}, \operatorname{div}\left(\widetilde{\boldsymbol{\sigma}}_{p}-\widetilde{\boldsymbol{\sigma}}_{q}\right)\right)_{\Omega},
$$

that $\left\{\widetilde{\boldsymbol{\sigma}}_{n}\right\}_{n}$ is a Cauchy sequence in $L^{2}(\Omega, \mathbb{M})$. Moreover, the identity

$$
\left(\mathcal{A}\left(\widetilde{\boldsymbol{\sigma}}_{p}-\widetilde{\boldsymbol{\sigma}}_{q}\right), \boldsymbol{\tau}\right)_{\Omega}+\left(\boldsymbol{\tau}, \widetilde{\boldsymbol{r}}_{p}-\widetilde{\boldsymbol{r}}_{q}\right)_{\Omega}=0, \quad \forall \boldsymbol{\tau} \in H\left(\operatorname{div}^{0}, \Omega, \mathbb{M}\right)
$$

and the inf-sup condition (6.3) yield

$$
\left\|\widetilde{\boldsymbol{r}}_{p}-\widetilde{\boldsymbol{r}}_{q}\right\|_{0, \Omega} \leq\|\Theta\| \sup _{\boldsymbol{\tau} \in H\left(\mathbf{d i v}^{0}, \Omega, \mathbb{M}\right)} \frac{\left(\boldsymbol{\tau}, \widetilde{\boldsymbol{r}}_{p}-\widetilde{\boldsymbol{r}}_{q}\right)_{\Omega}}{\|\boldsymbol{\tau}\|_{0, \Omega}} \leq\|\Theta\|\left\|\mathcal{A}\left(\widetilde{\boldsymbol{\sigma}}_{p}-\widetilde{\boldsymbol{\sigma}}_{q}\right)\right\|_{0, \Omega} .
$$

The last estimate ensures that $\left\{\widetilde{\boldsymbol{r}}_{n}\right\}_{n}$ is also a Cauchy sequence in $L^{2}(\Omega, \mathbb{M})$. We then come to the conclusion that the image under $P$ of any bounded sequence in $X$ contains a converging subsequence in $H$, which proves that Assumption 2 (i) is satisfieded.

On the other hand, testing (2.2) with $(\boldsymbol{\tau}, 0)$ and choosing the components of $\boldsymbol{\tau}: \Omega \rightarrow \mathbb{M}$ indefinitely differentiable and compactly supported in $\Omega$, we readily obtain that, if $\left(\sigma^{*}, \boldsymbol{r}^{*}\right):=$ $T((\sigma, r))$ then

$$
\nabla\left(\varrho^{-1} \operatorname{div} \boldsymbol{\sigma}^{*}\right)=\mathcal{A}\left(\boldsymbol{\sigma}^{*}-\boldsymbol{\sigma}\right)+\boldsymbol{r}^{*}-\boldsymbol{r} \in L^{2}(\Omega, \mathbb{M})
$$

Consequently,

$$
T(X) \cap P(X) \subset\left\{(\boldsymbol{\sigma}, \boldsymbol{r}) \in P(X) ; \varrho^{-1} \operatorname{div} \boldsymbol{\sigma} \in H^{1}\left(\Omega, \mathbb{R}^{d}\right)\right\},
$$


and the compactness of the embedding $T(X) \cap P(X) \hookrightarrow X$ follows. We conclude that Assumption 2 (ii) is fulfilled.

Finally, we notice that for all $0 \neq(\sigma, r) \in P(X)$,

$$
A((\boldsymbol{\sigma}, \boldsymbol{r}),(\boldsymbol{\sigma}, \boldsymbol{r})) \geq B((\boldsymbol{\sigma}, \boldsymbol{r}),(\boldsymbol{\sigma}, \boldsymbol{r}))=(\mathcal{A} \boldsymbol{\sigma}, \boldsymbol{\sigma})_{\Omega}>0,
$$

where we used that $\sigma$ is symmetric and that $\{0\} \times L^{2}(\Omega, \mathbb{K}) \subset K$.

We can invoke now Theorem 6.1 and Remark 2.1 to conclude that we have the following spectral characterization for the source operator $T$.

Proposition 6.1 The spectrum $\operatorname{sp}(T)$ of $T$ is given by $\operatorname{sp}(T)=\{0,1\} \cup\left\{\eta_{k}\right\}_{k \in \mathbb{N}}$, where $\left\{\eta_{k}\right\}_{k} \subset(0,1)$ is a sequence of finite-multiplicity eigenvalues of $T$ that converges to 0 . The ascent of each of these eigenvalues is 1 and the corresponding eigenfunctions lie in $P(X)$. Moreover, $\eta=1$ is an infinite-multiplicity eigenvalue of $T$ with associated eigenspace $K$ and $\eta=0$ is not an eigenvalue.

\subsubsection{The discrete problem}

We consider a family $\left\{\mathcal{T}_{h}\right\}_{h}$ of shape regular simplicial meshes of $\bar{\Omega}$ satisfying the standard finite element conformity assumptions. We denote by $h_{K}$ the diameter of triangles/tetrahedra

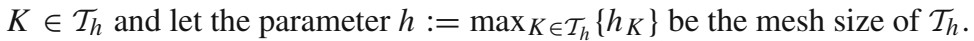

Hereafter, given an integer $m \geq 0$ and $D \subset \mathbb{R}^{d}, \mathcal{P}_{k}(D, E)$ is the space of functions with domain $D$ and values in $\mathrm{E}$, where $E$ is either $\mathbb{R}^{d}, \mathbb{M}$ or $\mathbb{K}$, and whose scalar components are polynomials of degree at most $m$. Likewise, the spaces of $E$-valued functions with piecewise polynomial scalar components of degree $\leq m$ relatively to $\mathcal{T}_{h}$ are defined by

$$
\mathcal{P}_{m}\left(\mathcal{T}_{h}, E\right):=\left\{\boldsymbol{v} \in L^{2}(\Omega, E) ;\left.\boldsymbol{v}\right|_{K} \in \mathcal{P}_{m}(K, E)\right\}, \quad \text { for } E=\mathbb{R}^{d}, \mathbb{M} \text { or } \mathbb{K} .
$$

For $k \geq 0$, we define Problem (3.1) with $X_{h}:=\mathcal{W}_{h} \times \mathcal{P}_{k}\left(\mathcal{T}_{h}, \mathbb{K}\right)$, where $\mathcal{W}_{h}:=$ $\mathcal{P}_{k+1}\left(\mathcal{T}_{h}, \overline{\mathbb{M}}\right) \cap H(\mathbf{d i v}, \Omega, \mathbb{M})$. We point out that the set $\left\{\mathcal{W}_{h}, \mathcal{P}_{k}\left(\mathcal{T}_{h}, \mathbb{R}^{d}\right), \mathcal{P}_{k}\left(\mathcal{T}_{h}, \mathbb{K}\right)\right\}$ constitutes the mixed finite element of Arnold-Falk-Winther [5] for linear elasticity. The key property ensuring the stability of this triplet of spaces is given by the following result, c.f. [5, Theorem 7.1].

Lemma 6.3 There exists a linear operator $\Theta_{h}: \mathcal{P}_{k}\left(\mathcal{T}_{h}, \mathbb{R}^{d}\right) \times \mathcal{P}_{k}\left(\mathcal{T}_{h}, \mathbb{K}\right) \rightarrow \mathcal{W}_{h}$ that is uniformly bounded with respect to $h$ and that satisfies

$$
\left(\operatorname{div} \Theta_{h}\left(\boldsymbol{v}_{h}, \boldsymbol{s}_{h}\right), \boldsymbol{v}_{h}\right)_{\Omega}+\left(\Theta_{h}\left(\boldsymbol{v}_{h}, \boldsymbol{s}_{h}\right), \boldsymbol{s}_{h}\right)_{\Omega}=\left\|\boldsymbol{v}_{h}\right\|_{0, \Omega}^{2}+\left\|\boldsymbol{s}_{h}\right\|_{0, \Omega}^{2}
$$

for all $\left(\boldsymbol{v}_{h}, \boldsymbol{s}_{h}\right) \in \mathcal{P}_{k}\left(\mathcal{T}_{h}, \mathbb{R}^{d}\right) \times \mathcal{P}_{k}\left(\mathcal{T}_{h}, \mathbb{K}\right)$.

We point out that $K_{h}=\mathcal{W}_{h}^{0} \times \mathcal{P}_{k}\left(\mathcal{T}_{h}, \mathbb{K}\right) \subset K$ where $\mathcal{W}_{h}^{0}:=\mathcal{W}_{h} \cap H\left(\operatorname{div}^{0}, \Omega, \mathbb{M}\right)$. The same procedure used in the proof of Corollary 6.1 can be used verbatim to deduce from Lemma 6.3 that Assumption 4 is satisfied. Moreover, as $\{0\} \times \mathcal{P}_{k}\left(\mathcal{T}_{h}, \mathbb{K}\right) \subset K_{h}$, for all $0 \neq\left(\boldsymbol{\sigma}_{h}, \boldsymbol{r}_{h}\right) \in P_{h}\left(X_{h}\right)=K_{h}^{\perp_{B}}$ it holds

$$
B\left(\left(\sigma_{h}, \boldsymbol{r}_{h}\right),\left(\mathbf{0}, \boldsymbol{s}_{h}\right)\right)=\left(\boldsymbol{\sigma}_{h}, \boldsymbol{s}_{h}\right)_{\Omega}=0, \quad \forall \boldsymbol{s}_{h} \in \mathcal{P}_{k}\left(\mathcal{T}_{h}, \mathbb{K}\right) .
$$

Consequently, the discrete counterpart of (6.6) is satisfied. Indeed, for all $0 \neq\left(\sigma_{h}, \boldsymbol{r}_{h}\right) \in$ $P_{h}\left(X_{h}\right)$ we have that

$$
A\left(\left(\sigma_{h}, \boldsymbol{r}_{h}\right),\left(\sigma_{h}, \boldsymbol{r}_{h}\right)\right) \geq B\left(\left(\boldsymbol{\sigma}_{h}, \boldsymbol{r}_{h}\right),\left(\sigma_{h}, \boldsymbol{r}_{h}\right)\right)=\left(\mathcal{A} \boldsymbol{\sigma}_{h}, \boldsymbol{\sigma}_{h}\right)_{\Omega}>0 .
$$

We deduce from Theorem 3.1 and Remark 3.1 the following result. 
Proposition 6.2 The spectrum of $T_{h}$ consists of $m:=\operatorname{dim}\left(X_{h}\right)$ eigenvalues, repeated accordingly to their respective multiplicities. It holds $\operatorname{sp}\left(T_{h}\right)=\{1\} \cup\left\{\eta_{h k}\right\}_{k=1}^{m_{0}}$, with $m_{0}=m-\operatorname{dim}\left(K_{h}\right)$. The eigenspace associated to $\eta_{h}=1$ is $K_{h}$. The real numbers $\eta_{h k} \in(0,1), k=1, \ldots, m_{0}$, are non-defective eigenvalues with eigenspaces lying in $K_{h}^{\perp_{B}}$ and $\eta_{h}=0$ is not an eigenvalue.

Let us now recall some well-known approximation properties of the finite element spaces introduced above. Given $s>1 / 2$ the tensorial version of the canonical interpolation operator $\Pi_{h}: H^{s}(\Omega, \mathbb{M}) \rightarrow \mathcal{W}_{h}$ associated with the Brezzi-Douglas-Marini (BDM) mixed finite element [12], satisfies the following classical error estimate, see [11, Proposition 2.5.4],

$$
\left\|\boldsymbol{\tau}-\Pi_{h} \boldsymbol{\tau}\right\|_{0, \Omega} \leq C h^{\min \{s, k+2\}}\|\boldsymbol{\tau}\|_{s, \Omega} \quad \forall \boldsymbol{\tau} \in H^{s}(\Omega, \mathbb{M}), \quad s>1 / 2,
$$

Moreover, we have the well-known commutativity property,

$$
\operatorname{div} \Pi_{h} \sigma=U_{h} \operatorname{div} \sigma, \quad \forall \sigma \in H^{s}(\Omega, \mathbb{M}) \cap H(\operatorname{div}, \Omega, \mathbb{M}), \quad s>1 / 2 .
$$

where $U_{h}$ stands for the $L^{2}\left(\Omega, \mathbb{R}^{d}\right)$-orthogonal projection onto $\mathcal{P}_{k}\left(\mathcal{T}_{h}, \mathbb{R}^{d}\right)$. Therefore, if $\operatorname{div} \tau \in H^{s}\left(\Omega, \mathbb{R}^{d}\right)$, we obtain

$$
\left\|\operatorname{div}\left(\boldsymbol{\tau}-\Pi_{h} \boldsymbol{\tau}\right)\right\|_{0, \Omega}=\left\|\operatorname{div} \boldsymbol{\tau}-U_{h} \operatorname{div} \boldsymbol{\tau}\right\|_{0, \Omega} \leq C h^{\min \{s, k+1\}}\|\operatorname{div} \boldsymbol{\tau}\|_{s, \Omega} .
$$

Finally, if $S_{h}$ represents the $L^{2}(\Omega, \mathbb{M})$-orthogonal projection onto $\mathcal{P}_{k}\left(\mathcal{T}_{h}, \mathbb{K}\right)$, for any $s>0$, it holds

$$
\left\|\boldsymbol{s}-S_{h} \boldsymbol{s}\right\|_{0, \Omega} \leq C h^{\min \{s, k+1\}}\|\boldsymbol{s}\|_{s, \Omega} \quad \forall s \in H^{s}(\Omega, \mathbb{K}) .
$$

We point out that one can actually extend the domain of the canonical interpolation operator $\Pi_{h}$ to $H(\operatorname{div}, \Omega, \mathbb{M}) \cap H^{s}(\Omega, \mathbb{M})$, for any $s>0$. In the case of a constant function $\varrho$ and a constant tensor $\mathcal{A}$, classical regularity results [15,22] ensure the existence of $\hat{s} \in(0,1]$ (depending on $\Omega$ on the boundary conditions and on the Lamé coefficients) such that the solution $\tilde{\boldsymbol{u}}$ of problem (6.4) belongs to $H^{1+s}\left(\Omega, \mathbb{R}^{d}\right) \cap H_{0}^{1}\left(\Omega, \mathbb{R}^{d}\right)$ for all $s \in(0, \hat{s})$. This implies that $P(X) \subset\left[H^{s}(\Omega, \mathbb{M}) \cap H(\operatorname{div}, \Omega, \mathbb{M})\right] \times H^{s}(\Omega, \mathbb{M})$. In such a case, we can directly define the operator linear operator $\Xi_{h}: K^{\perp_{B}} \rightarrow X_{h}$ by $\Xi_{h}(\sigma, r):=\left(\Pi_{h} \boldsymbol{\sigma}, S_{h} \boldsymbol{r}\right)$ and deduce (as shown below) that Assumption 5 is satisfied. However, instead of relying on regularity results that are difficult to establish for the elasticity system in the case of general domains, boundary conditions and material properties, we resort to the quasi-interpolation operator constructed in $[13,19,25]$ by combining the BDM interpolation operator $\Pi_{h}$ with a mollification technique. The resulting projector has domain $H(\mathbf{d i v}, \Omega, \mathbb{M})$ and range $\mathcal{W}_{h}$ and preserves all the properties of $\Pi_{h}$ listed above. More precisely, we will use tensorial version of the following result [19, Theorem 6.5] (see also [13,25]).

Theorem 6.1 There exists a bounded and linear operator $\mathcal{J}_{h}: H(\operatorname{div}, \Omega, \mathbb{M}) \rightarrow \mathcal{W}_{h}$ such that

(i) $\mathcal{W}_{h}$ is point-wise invariant under $\mathcal{J}_{h}$

(ii) The exists $C>0$ independent of $h$ such that

$$
\left\|\boldsymbol{\sigma}-\mathcal{J}_{h} \boldsymbol{\sigma}\right\|_{0, \Omega} \leq C \inf _{\tau_{h} \in \mathcal{W}_{h}}\left\|\boldsymbol{\sigma}-\boldsymbol{\tau}_{h}\right\|_{0, \Omega}, \quad \forall \boldsymbol{\sigma} \in H(\operatorname{div}, \Omega, \mathbb{M})
$$

iii) $\operatorname{div} \mathcal{J}_{h} \sigma=U_{h} \operatorname{div} \sigma$ for all $\sigma \in H(\operatorname{div}, \Omega, \mathbb{M})$.

Lemma 6.4 The linear operator $\Xi_{h}: X \rightarrow X_{h}$ defined by $\Xi_{h}(\boldsymbol{\sigma}, \boldsymbol{r}):=\left(\mathcal{J}_{h} \boldsymbol{\sigma}, S_{h} \boldsymbol{r}\right)$ satisfies Assumption 5. 
Proof For an arbitrary $\left(\boldsymbol{\sigma}_{h}, \boldsymbol{r}_{h}\right) \in X_{h}$ we let $(\widetilde{\boldsymbol{\sigma}}, \widetilde{\boldsymbol{r}})=P\left(\boldsymbol{\sigma}_{h}, \boldsymbol{r}_{h}\right) \in K^{\perp_{B}}$. It holds

$$
\left(\boldsymbol{\sigma}_{h}, \boldsymbol{r}_{h}\right)-\Xi_{h} P\left(\sigma_{h}, \boldsymbol{r}_{h}\right)=\left(\sigma_{h}-\mathcal{J}_{h} \tilde{\boldsymbol{\sigma}}, \boldsymbol{r}_{h}-S_{h} \tilde{\boldsymbol{r}}\right) \in K_{h}=\mathcal{W}_{h}^{0} \times \mathcal{P}_{k}\left(\mathcal{T}_{h}, \mathbb{K}\right) .
$$

Indeed, by virtue of property (iii) of Theorem 6.1 and because $\operatorname{div} \widetilde{\sigma}=\operatorname{div} \sigma_{h}$, we have that

$$
\operatorname{div}\left(\sigma_{h}-\mathcal{J}_{h} \tilde{\sigma}\right)=\operatorname{div} \sigma_{h}-U_{h} \operatorname{div} \tilde{\sigma}=\operatorname{div} \sigma_{h}-U_{h} \operatorname{div} \sigma_{h}=0 .
$$

This proves Assumption 5 (iii). On the other hand, using this time property (ii) of Theorem 6.1, we deduce that, if we let $(\widetilde{\boldsymbol{\sigma}}, \tilde{\boldsymbol{r}})=P(\boldsymbol{\sigma}, \boldsymbol{r}) \in K^{\perp_{B}}$ for an arbitrary $(\boldsymbol{\sigma}, \boldsymbol{r}) \in X$, we obtain

$$
\begin{aligned}
\left\|\left(I-\Xi_{h}\right) P(\boldsymbol{\sigma}, \boldsymbol{r})\right\|_{H}^{2} & =\left\|\tilde{\boldsymbol{\sigma}}-\mathcal{J}_{h} \tilde{\boldsymbol{\sigma}}\right\|_{0, \Omega}^{2}+\left\|\tilde{\boldsymbol{r}}-S_{h} \widetilde{\boldsymbol{r}}\right\|_{0, \Omega}^{2} \\
& \lesssim \inf _{\boldsymbol{\tau}_{h} \in \mathcal{W}_{h}}\left\|\tilde{\boldsymbol{\sigma}}-\boldsymbol{\tau}_{h}\right\|_{0, \Omega}^{2}+\left\|\tilde{\boldsymbol{r}}-S_{h} \widetilde{\boldsymbol{r}}\right\|_{0, \Omega}^{2},
\end{aligned}
$$

and again by property (iii) of Theorem 6.1

$$
\begin{aligned}
\left\|\left(I-\Xi_{h}\right) P(\boldsymbol{\sigma}, \boldsymbol{r})\right\|_{X}^{2} & =\left\|\left(I-\Xi_{h}\right) P(\boldsymbol{\sigma}, \boldsymbol{r})\right\|_{H}^{2}+\left\|\operatorname{div}\left(\tilde{\boldsymbol{\sigma}}-\mathcal{J}_{h} \tilde{\boldsymbol{\sigma}}\right)\right\|_{0, \Omega}^{2} \\
& \lesssim \inf _{\boldsymbol{\tau}_{h} \in \mathcal{W}_{h}}\left\|\tilde{\boldsymbol{\sigma}}-\boldsymbol{\tau}_{h}\right\|_{0, \Omega}^{2}+\left\|\operatorname{div} \tilde{\boldsymbol{\sigma}}-U_{h} \operatorname{div} \tilde{\boldsymbol{\sigma}}\right\|_{0, \Omega}^{2}+\left\|\widetilde{\boldsymbol{r}}-S_{h} \tilde{\boldsymbol{r}}\right\|_{0, \Omega}^{2}
\end{aligned}
$$

It follows immediately from (6.12)-(6.13) and the triangle inequality that Assumption 5 (i) is satisfied. Moreover, the error estimates (6.8), (6.10) and (6.11) and classical density results ensure that Assumption 5 (ii) is a consequence of (6.13).

We conclude that the Galerkin method (3.1) provides a correct spectral approximation of the eigenproblem (6.1) in the sense of Theorem 4.1.

We recall that $\eta \in(0,1)$ is an eigenvalue of $T$ with multiplicity $m$ if and only if $\kappa=1 / \eta$ is an eigenvalue of Problem 2.1 with the same multiplicity and the corresponding eigenfunctions coincide. Analogously, $\eta_{i, h}, i=1, \ldots, m$, are the eigenvalues of $T_{h}$ (repeated accordingly to their respective multiplicities) that converge to $\eta$ if and only if $\kappa_{i, h}=1 / \eta_{i, h}$ are the eigenvalues of Problem 3.1 converging to $\eta$. Moreover, the corresponding eigenfunctions coincide. Taking into account that Assumption 6 is satisfied because of (6.6), the following rates of convergence for the eigenfunctions and eigenvalues are a direct consequence of Theorem 5.1, (6.6) and Theorem 5.2, together with the interpolation error estimates (6.8), (6.10) and (6.11).

Theorem 6.2 Let $\eta \neq 1$ be an eigenvalue of $T$ of algebraic multiplicity $m$ and let $\mathcal{E}_{\eta}(X)$ be the corresponding eigenspace. There exists $h_{0}>0$ such that for all $h \leq h_{0}, T_{h}$ admits exactly m eigenvalues $\eta_{i, h}, i=1, \ldots, m$, repeated according to their respective multiplicity, such that

$$
\lim _{h \rightarrow 0} \widehat{\delta}\left(\mathcal{E}_{\eta}(X), \mathcal{E}_{\eta, h}\left(X_{h}\right)\right)=0 \text { and } \lim _{h \rightarrow 0} \max _{1 \leq i \leq m}\left|\eta-\eta_{i, h}\right|=0,
$$

where $\mathcal{E}_{\eta, h}\left(X_{h}\right)$ is the $T_{h}$-invariant subspace of $X_{h}$ spanned by the eigenspaces of $\left\{\eta_{i, h}, i=\right.$ $1, \ldots, m\}$. Moreover, if we assume that the eigenfunctions are piecewise regular with respect to a partition $\bar{\Omega}=\cup_{j=1}^{J} \bar{\Omega}_{j}$ of $\Omega$ into polyhedral/polygonal subdomain $\Omega_{j}$, namely, if there exists $r>1 / 2$ such that

$$
\mathcal{E}_{\eta}(X) \subset\left\{(\boldsymbol{\tau}, \boldsymbol{s}) \in H^{r}\left(\cup_{j=1}^{J} \Omega_{j}, \mathbb{M}\right) \times H^{r}\left(\cup_{j=1}^{J} \Omega_{j}, \mathbb{K}\right) ; \operatorname{div} \boldsymbol{\tau} \in H^{r}\left(\cup_{j=1}^{J} \Omega_{j}, \mathbb{R}^{d}\right)\right\},
$$

then, for $h$ small enough,

$$
\widehat{\delta}\left(\mathcal{E}_{\eta}(X), \mathcal{E}_{\eta, h}\left(X_{h}\right)\right) \lesssim h^{\min \{r, k+1\}} \text { and } \max _{1 \leq i \leq m}\left|\kappa-\kappa_{i, h}\right| \lesssim\left(h^{\min \{r, k+1\}}\right)^{2} .
$$


Remark 6.1 For the sake of brevity and clarity of exposition, we only considered here an approximation based on the Arnold-Falk-Winther element [5]. However, we could equally have defined the Galerkin method in base of the families of mixed finite elements introduced by Cockburn-Gopalakrishnan-Guzmán $(\mathrm{CGG}(k))$ in [14] and by Gopalakrishnan-Guzmán $(\mathrm{GG}(k))$ in [21] for $k \geq 1$. Indeed, the same BDM quasi-interpolation operator $\mathcal{J}_{h}$ can be used in the proof of Assumption 5 for $\mathrm{GG}(k)$ and the quasi-interpolation operator corresponding to the Raviart-Thomas element can be used instead for CGG $(k)$.

\subsection{Stress formulation of the Stokes eigenproblem with reduced symmetry}

\subsubsection{The continuous problem}

We are interested in the following eigenvalue problem for the Stokes system posed in a connected Lipschitz domain $\Omega \subset \mathbb{R}^{d}$ : find $(\lambda, \boldsymbol{u}, p)$, with $\boldsymbol{u} \neq 0$ and $\lambda \in \mathbb{R}$ such that,

$$
\begin{aligned}
-\operatorname{div}(\varepsilon(u)-p I) & =\lambda \boldsymbol{u} \quad \text { in } \Omega, \\
\operatorname{div} \boldsymbol{u} & =0 \quad \text { in } \Omega, \\
\boldsymbol{u} & =0 \text { on } \partial \Omega .
\end{aligned}
$$

It is interesting to notice that, in the two dimensional case, problem (6.15) is equivalent to the following buckling problem for a uniformly compressed clamped Kirchhoff plate (see [29]): find $\lambda \in \mathbb{R}$ and $0 \neq \psi \in H_{0}^{2}(\Omega)$ such that

$$
\begin{aligned}
-\Delta^{2} \psi & =2 \lambda \Delta \psi, \quad \text { in } \Omega, \\
\psi & =\nabla \psi \cdot \boldsymbol{n}=0 \quad \text { on } \partial \Omega,
\end{aligned}
$$

where $\boldsymbol{n}$ represents the unit outward normal vector to $\partial \Omega$. The eigenvalue $2 \lambda$ represents in this case the buckling coefficient. The plate transverse displacement $\psi$ is related to the Stokes velocity field by $\frac{1}{2} \boldsymbol{u}=\operatorname{curl} \psi:=\left(\partial_{2} \psi,-\partial_{1} \psi\right)^{\mathrm{t}}$.

Our aim is to employ a dual-mixed approach to derive a variational formulation of this problem. To this end, we introduce the stress tensor $\sigma:=\boldsymbol{\varepsilon}(\boldsymbol{u})-p I$ and notice that the incompressibility condition implies that $\boldsymbol{\sigma}^{\mathrm{D}}=\nabla \boldsymbol{u}-\boldsymbol{r}$, with $\boldsymbol{r}:=\frac{1}{2}\left[\nabla \boldsymbol{u}-(\nabla \boldsymbol{u})^{\mathrm{t}}\right]$. We can then reformulate the problem above in terms of $\sigma$ as follows: find $\lambda \in \mathbb{R}, 0 \neq \sigma: \Omega \rightarrow \mathbb{S}$, and $0 \neq \boldsymbol{r} \rightarrow \mathbb{K}$ such that,

$$
\begin{aligned}
-\nabla(\operatorname{div} \sigma) & =\lambda\left(\boldsymbol{\sigma}^{\mathrm{D}}+\boldsymbol{r}\right) \quad \text { in } \Omega, \\
\operatorname{div} \sigma & =0 \quad \text { on } \partial \Omega .
\end{aligned}
$$

We point out that the pressure $p$ and the velocity field $\boldsymbol{u}$ disappeared from the formulation but once $\sigma$ is known they can be recovered (and also post-processed at the discrete level) by $\boldsymbol{u}=-\frac{1}{\lambda} \operatorname{div} \sigma$ and $p=-\frac{1}{d} \operatorname{tr}(\sigma)$.

The variational formulation (6.16) fits into the abstract framework of Sect. 2 by taking in problem $(2.1) \kappa=1+\lambda, H:=L^{2}(\Omega, \mathbb{M}) \times L^{2}(\Omega, \mathbb{M}), X:=H(\operatorname{div}, \Omega, \mathbb{M}) \times L^{2}(\Omega, \mathbb{K})$ (endowed with their natural norms (6.2)) and by defining the bounded and symmetric bilinear forms $B: H \times H \rightarrow \mathbb{R}, c: X \times X \rightarrow \mathbb{R}$ and $A: X \times X \rightarrow \mathbb{R}$ as follows:

$$
\begin{aligned}
B((\boldsymbol{\sigma}, \boldsymbol{r}),(\boldsymbol{\tau}, \boldsymbol{s})):= & \left(\boldsymbol{\sigma}^{\mathrm{D}}, \boldsymbol{\tau}^{\mathrm{D}}\right)_{\Omega}+(\operatorname{tr}(\boldsymbol{\sigma}), 1)_{\Omega}(\operatorname{tr}(\boldsymbol{\tau}), 1)_{\Omega}+(\boldsymbol{r}, \boldsymbol{\tau})_{\Omega}+(\boldsymbol{s}, \boldsymbol{\sigma})_{\Omega}, \\
c((\boldsymbol{\sigma}, \boldsymbol{r}),(\boldsymbol{\tau}, \boldsymbol{s})):= & (\operatorname{div} \boldsymbol{\sigma}, \operatorname{div} \boldsymbol{\tau})_{\Omega}, A((\boldsymbol{\sigma}, \boldsymbol{r}),(\boldsymbol{\tau}, \boldsymbol{s})):=c((\boldsymbol{\sigma}, \boldsymbol{r}),(\boldsymbol{\tau}, \boldsymbol{s})) \\
& +B((\boldsymbol{\sigma}, \boldsymbol{r}),(\boldsymbol{\tau}, \boldsymbol{s})) .
\end{aligned}
$$


We point out that testing problem (2.1) with $(I, 0) \in X$ gives $(\operatorname{tr}(\sigma), 1)_{\Omega}=0$ for $\kappa \neq 1$. This corresponds to the zero mean value restriction on the pressure $p \in L_{0}^{2}(\Omega):=\{q \in$ $\left.L^{2}(\Omega) ;(q, 1)_{\Omega}=0\right\}$ that is usually imposed to enforce uniqueness. Here, we opt for a variational insertion of this restriction in order to free the space $X$ from the cumbersome constraint $(\operatorname{tr}(\boldsymbol{\tau}), 1)_{\Omega}=0$.

The following Poincaré-Friedrichs inequality is essential in our analysis.

Lemma 6.5 There exists $\alpha>0$ depending only on $\Omega$ such that

$$
\alpha\|\boldsymbol{\tau}\|_{0, \Omega}^{2} \leq\left\|\boldsymbol{\tau}^{\mathrm{D}}\right\|_{0, \Omega}^{2}+\|\operatorname{div} \boldsymbol{\tau}\|_{0, \Omega}^{2}, \quad \forall \boldsymbol{\tau} \in H(\operatorname{div}, \Omega, \mathbb{M}), \quad(\operatorname{tr}(\boldsymbol{\tau}), 1)_{\Omega}=0 .
$$

Proof See [11, Proposition 9.1.1].

\section{Corollary 6.2 The bilinear form}

$$
(\boldsymbol{\sigma}, \boldsymbol{\tau}) \rightarrow\left(\boldsymbol{\sigma}^{\mathrm{D}}, \boldsymbol{\tau}^{\mathrm{D}}\right)_{\Omega}+(\operatorname{tr}(\boldsymbol{\sigma}), 1)_{\Omega}(\operatorname{tr}(\boldsymbol{\tau}), 1)_{\Omega}+(\operatorname{div} \boldsymbol{\sigma}, \operatorname{div} \boldsymbol{\tau})_{\Omega}
$$

is coercive on $H(\operatorname{div}, \Omega, \mathbb{M})$.

Proof Using the $L^{2}(\Omega, \mathbb{M})$-orthogonal decomposition $\tau=\tau_{0}+\frac{1}{d|\Omega|}(\operatorname{tr}(\tau), 1)_{\Omega} I$, we deduce from Lemma 6.5 that

$$
\begin{aligned}
&\|\boldsymbol{\tau}\|_{\operatorname{div}, \Omega}^{2}=\left\|\boldsymbol{\tau}_{0}\right\|_{0, \Omega}^{2}+\frac{1}{d|\Omega|}(\operatorname{tr}(\boldsymbol{\tau}), 1)_{\Omega}^{2}+\|\operatorname{div} \boldsymbol{\tau}\|_{0, \Omega}^{2} \\
& \leq \max \left\{1+\frac{1}{\alpha}, \frac{1}{d|\Omega|}\right\}\left(\left\|\boldsymbol{\tau}^{\mathrm{D}}\right\|_{0, \Omega}^{2}+(\operatorname{tr}(\boldsymbol{\tau}), 1)_{\Omega}^{2}+\|\operatorname{div} \boldsymbol{\tau}\|_{0, \Omega}^{2}\right), \\
& \forall \boldsymbol{\tau} \in H(\operatorname{div}, \Omega, \mathbb{M}),
\end{aligned}
$$

and the result follows.

We point out that Corollary 6.2 also implies the coerciveness of the bilinear form

$$
(\boldsymbol{\sigma}, \boldsymbol{\tau}) \rightarrow\left(\boldsymbol{\sigma}^{\mathrm{D}}, \boldsymbol{\tau}^{\mathrm{D}}\right)_{\Omega}+(\operatorname{tr}(\boldsymbol{\sigma}), 1)_{\Omega}(\operatorname{tr}(\boldsymbol{\tau}), 1)_{\Omega}
$$

on $H\left(\mathbf{d i v}^{0}, \Omega, \mathbb{M}\right)$. Consequently, following the same steps given in the proof of Corollary 6.1 we deduce that Assumption 1 is satisfied. Hence, the source operator $T: X \rightarrow X$ defined by problem (2.2) is bounded and symmetric with respect to $B(\cdot, \cdot)$. In addition, we have the direct and stable splitting $X=K \oplus K^{\perp_{B}}$, where $K:=H\left(\operatorname{div}^{0}, \Omega, \mathbb{M}\right) \times L^{2}(\Omega, \mathbb{K})$ is the eigenspace corresponding to the essential eigenvalue $\eta=1$ of (2.1). We also have that $T\left(K^{\perp_{B}}\right) \subset K^{\perp_{B}}$. It is important to notice that $T$ is not one-to-one. Indeed, if $(\sigma, \boldsymbol{r}) \in X$ is such that $B((\sigma, r),(\tau, s))=0$ for all $(\tau, s) \in X$ then $\sigma=\sigma^{\mathrm{t}},(\sigma, 1)_{\Omega}=0$ and

$$
\left(\boldsymbol{\sigma}^{\mathrm{D}}, \boldsymbol{\tau}\right)_{\Omega}+(\boldsymbol{r}, \boldsymbol{\tau})_{\Omega}=0, \quad \forall \boldsymbol{\tau} \in H(\operatorname{div}, \Omega, \mathbb{M}) .
$$

Taking $\boldsymbol{\tau}=\boldsymbol{\sigma}^{\mathrm{D}}$ in (6.18) we deduce that $\boldsymbol{\sigma}^{\mathrm{D}}=0$. This condition holds true if and only if $\sigma=p I$, with $p=\frac{1}{d} \operatorname{tr}(\sigma) \in L^{2}(\Omega)$ and $\nabla p=\operatorname{div} \sigma \in L^{2}\left(\Omega, \mathbb{R}^{d}\right)$. Testing now (6.18) with skew symmetric tensors and using a density argument we deduce that $\boldsymbol{r}=0$. It follows that

$$
\operatorname{ker}(T)=\left\{q I ; q \in H^{1}(\Omega) \cap L_{0}^{2}(\Omega)\right\} \times\{0\}
$$

is the eigenspace corresponding to the eigenvalue $\eta=0$ of $T$.

The characterization of the projector $P: X \rightarrow X$ associated to this decomposition follows the same pattern of last section. For any $(\boldsymbol{\sigma}, \boldsymbol{r}) \in X$, we consider $P(\boldsymbol{\sigma}, \boldsymbol{r})=(\widetilde{\boldsymbol{\sigma}}, \widetilde{\boldsymbol{r}}) \in X$ with $\tilde{\boldsymbol{\sigma}}:=\boldsymbol{\varepsilon}(\tilde{\boldsymbol{u}})-\tilde{p} I$ and $\tilde{\boldsymbol{r}}:=\frac{1}{2}\left[\nabla \tilde{\boldsymbol{u}}-(\nabla \tilde{\boldsymbol{u}})^{\mathrm{t}}\right]$, where $(\tilde{\boldsymbol{u}}, \tilde{p}) \in H_{0}^{1}\left(\Omega, \mathbb{R}^{d}\right) \times L_{0}^{2}(\Omega)$ is the 
unique solution of the classical velocity-pressure variational formulation of Stokes problem in $\Omega$ with load $-\operatorname{div} \sigma$, i.e.,

$$
\begin{aligned}
& (\boldsymbol{\varepsilon}(\tilde{\boldsymbol{u}}), \boldsymbol{\varepsilon}(\boldsymbol{v}))_{\Omega}-(\operatorname{div} \tilde{\boldsymbol{u}}, q)_{\Omega}-(\operatorname{div} \boldsymbol{v}, \tilde{p})_{\Omega} \\
& \quad=-(\operatorname{div} \boldsymbol{\sigma}, \boldsymbol{v})_{\Omega}, \quad \forall(\boldsymbol{v}, q) \in H_{0}^{1}\left(\Omega, \mathbb{R}^{d}\right) \times L_{0}^{2}(\Omega),
\end{aligned}
$$

We know [11] that there exists a constant $C>0$ such that

$$
\|\tilde{\boldsymbol{u}}\|_{1, \Omega}+\|\tilde{p}\|_{0, \Omega} \leq C\|\operatorname{div} \boldsymbol{\sigma}\|_{0, \Omega},
$$

moreover, $\operatorname{div} \widetilde{\sigma}=\operatorname{div} \sigma$ by construction. This ensures the continuity of $P: X \rightarrow X$. Besides, it is clear that $P \circ P=P$, ker $P=K$, and for any $(\sigma, r) \in X$,

$$
B(P(\boldsymbol{\sigma}, \boldsymbol{r}),(\boldsymbol{\tau}, \boldsymbol{s}))=(\nabla \tilde{\boldsymbol{u}}, \boldsymbol{\tau})_{\Omega}=0, \quad \forall(\boldsymbol{\tau}, \boldsymbol{s}) \in K,
$$

which proves that $P(X) \subset K^{\perp_{B}}$. The inclusion $K^{\perp_{B}} \subset P(X)$ is a consequence of $(I-$ $P) X \subset K$. We conclude that $P: X \rightarrow X$ is the unique continuous projector associated to the direct and stable decomposition $X=K \oplus K^{\perp_{B}}$.

Lemma 6.6 Assumption 2 is satisfied.

Proof Let $\left\{\left(\sigma_{n}, \boldsymbol{r}_{n}\right)\right\}_{n}$ be a weakly convergent sequence in $X$. The continuity of $P: X \rightarrow X$ implies that $\left\{\left(\widetilde{\boldsymbol{\sigma}}_{n}, \widetilde{\boldsymbol{r}}_{n}\right)\right\}_{n}:=\left\{P\left(\boldsymbol{\sigma}_{n}, \boldsymbol{r}_{n}\right)\right\}_{n}=\left\{\left(\nabla \tilde{\boldsymbol{u}}_{n}-\tilde{p}_{n} I, \frac{1}{2}\left[\nabla \tilde{\boldsymbol{u}}_{n}-\left(\nabla \tilde{\boldsymbol{u}}_{n}\right)^{\mathrm{t}}\right]\right)\right\}_{n}$ also converges weakly in $X$, where $\left(\tilde{\boldsymbol{u}}_{n}, \tilde{p}_{n}\right) \in H_{0}^{1}\left(\Omega, \mathbb{R}^{d}\right) \times L_{0}^{2}(\Omega)$ solves (6.19) with datum $-\operatorname{div} \sigma_{n}$. As a consequence of $(6.20),\left\{\tilde{\boldsymbol{u}}_{n}\right\}_{n}$ is bounded in $H^{1}\left(\Omega, \mathbb{R}^{d}\right)$. In turn, we deduce from the identities $-\nabla \tilde{p}_{n}=\operatorname{div} \widetilde{\sigma}_{n}=\operatorname{div} \sigma_{n}$ that $\left\{\tilde{p}_{n}\right\}_{n}$ is also bounded in $H^{1}(\Omega)$. The compactness of the embedding $H^{1}\left(\Omega, \mathbb{R}^{d}\right) \times H^{1}(\Omega) \hookrightarrow L^{2}\left(\Omega, \mathbb{R}^{d}\right) \times L^{2}(\Omega)$ imply that $\left\{\tilde{\boldsymbol{\sigma}}_{n}, \tilde{p}_{n}\right\}_{n}$ admits a subsequence (also denotes $\left\{\tilde{\boldsymbol{\sigma}}_{n}, \tilde{p}_{n}\right\}_{n}$ ) that converges strongly in $L^{2}\left(\Omega, \mathbb{R}^{d}\right) \times L^{2}(\Omega)$. Next, it follows from (6.19) and a Green formula that

$\left(\boldsymbol{\varepsilon}\left(\tilde{\boldsymbol{u}}_{p}-\tilde{\boldsymbol{u}}_{q}\right), \boldsymbol{\varepsilon}(\boldsymbol{v})\right)_{\Omega}-\left(\operatorname{div} \boldsymbol{v}, \tilde{p}_{p}-\tilde{p}_{q}\right)_{\Omega}=-\left(\operatorname{div}\left(\boldsymbol{\sigma}_{p}-\boldsymbol{\sigma}_{q}\right), v\right)_{\Omega}, \quad \forall \boldsymbol{v} \in H_{0}^{1}\left(\Omega, \mathbb{R}^{d}\right)$.

Taking $\boldsymbol{v}=\tilde{\boldsymbol{u}}_{p}-\tilde{\boldsymbol{u}}_{q}$ in the last identity yields

$$
\left\|\boldsymbol{\varepsilon}\left(\tilde{\boldsymbol{u}}_{p}-\tilde{\boldsymbol{u}}_{q}\right)\right\|_{0, \Omega}^{2}=-\left(\operatorname{div}\left(\sigma_{p}-\sigma_{q}\right), \tilde{\boldsymbol{u}}_{p}-\tilde{\boldsymbol{u}}_{q}\right)_{\Omega},
$$

which proves that $\left\{\boldsymbol{\varepsilon}\left(\tilde{\boldsymbol{u}}_{n}\right)\right\}_{n}$ is a Cauchy sequence in $L^{2}(\Omega, \mathbb{M})$. Moreover, testing the identity $\nabla\left(\boldsymbol{u}_{p}-\boldsymbol{u}_{q}\right)=\left(\widetilde{\boldsymbol{\sigma}}_{p}-\widetilde{\boldsymbol{\sigma}}_{q}\right)^{\mathrm{D}}+\widetilde{\boldsymbol{r}}_{p}-\tilde{\boldsymbol{r}}_{q}$ with $\boldsymbol{\tau} \in H\left(\operatorname{div}^{0}, \Omega, \mathbb{M}\right)$ yields

$$
\left(\left(\widetilde{\boldsymbol{\sigma}}_{p}-\widetilde{\boldsymbol{\sigma}}_{q}\right)^{\mathrm{D}}, \boldsymbol{\tau}^{\mathrm{D}}\right)_{\Omega}+\left(\widetilde{\boldsymbol{r}}_{p}-\widetilde{\boldsymbol{r}}_{q}, \boldsymbol{\tau}\right)_{\Omega}=0, \quad \forall \boldsymbol{\tau} \in H\left(\mathbf{d i v}^{0}, \Omega, \mathbb{M}\right) .
$$

We can now use the inf-sup condition (6.3) as in Lemma 6.2 to deduce that $\left\{\widetilde{\boldsymbol{r}}_{n}\right\}_{n}$ is also a Cauchy sequence in $L^{2}(\Omega, \mathbb{M})$. We conclude that $\left\{\left(\widetilde{\boldsymbol{\sigma}}_{n}, \widetilde{\boldsymbol{r}}_{n}\right)\right\}_{n}:=\left\{P\left(\boldsymbol{\sigma}_{n}, \boldsymbol{r}_{n}\right)\right\}_{n}$ admits a subsequence that converges strongly in $H$ and Assumption 2 (i) follows.

Let us denote by $\left(\sigma^{*}, r^{*}\right):=T(\sigma, r)$ the image of any $(\sigma, r) \in X$ by the source operator $T$. Testing (2.2) with $(I, 0) \in X$ we deduce that $\left(\sigma^{*}, 1\right)_{\Omega}=(\sigma, 1)_{\Omega}$. Testing now the same equation with $(\boldsymbol{\tau}, 0)$, and choosing the entries of $\boldsymbol{\tau}: \Omega \rightarrow \mathbb{M}$ indefinitely differentiable and compactly supported in $\Omega$, we deduce that

$$
\nabla\left(\operatorname{div} \sigma^{*}\right)=\left(\sigma^{*}-\sigma\right)^{\mathrm{D}}+\boldsymbol{r}^{*}-\boldsymbol{r} \in L^{2}(\Omega, \mathbb{M})
$$

Therefore, the subspace

$$
T(X) \cap P(X) \subset\left\{(\boldsymbol{\sigma}, \boldsymbol{r}) \in P(X) ; \operatorname{div} \boldsymbol{\sigma} \in H^{1}\left(\Omega, \mathbb{R}^{d}\right)\right\},
$$

is compactly embedded in $X$ and Assumption 2 (ii) is also satisfied. 
Before we provide the spectral description of $T$ given by Theorem 6.1, we point out that for $\eta \notin\{0,1\}$ is an eigenvalue of $T$ with eigenspace $\mathcal{E}_{\eta}(X)$ then

$$
A((\boldsymbol{\sigma}, \boldsymbol{r}),(\boldsymbol{\sigma}, \boldsymbol{r})) \geq B((\boldsymbol{\sigma}, \boldsymbol{r}),(\boldsymbol{\sigma}, \boldsymbol{r}))=\left\|\boldsymbol{\sigma}^{\mathrm{D}}\right\|_{0, \Omega}^{2}>0, \quad \forall(\boldsymbol{\sigma}, \boldsymbol{r}) \in \mathcal{E}_{\eta}(X)
$$

where we used that $\sigma$ is symmetric and satisfies the restriction $(\sigma, 1)_{\Omega}=0$ together with the fact that $\{0\} \times L^{2}(\Omega, \mathbb{K}) \subset K$ and $\mathcal{E}_{\eta}(X) \not \subset \operatorname{ker}(T)$.

Proposition 6.3 The spectrum $\operatorname{sp}(T)$ of T admits the decomposition $\operatorname{sp}(T)=\{0,1\} \cup\left\{\eta_{k}\right\}_{k \in \mathbb{N}}$, where $\left\{\eta_{k}\right\}_{k} \subset(0,1)$ is a sequence of finite-multiplicity eigenvalues of $T$ that converges to 0 . The ascent of each of these eigenvalues is 1 and the corresponding eigenfunctions lie in $P(X)$. Moreover, $\eta=1$ is an infinite-multiplicity eigenvalue of $T$ with associated eigenspace $K$ and $\eta=0$ is an eigenvalue of with eigenspace $\operatorname{ker}(T)$.

\subsubsection{The discrete problem}

The Galerkin scheme (3.1) is based on the same finite element spaces used in the last section. Namely, for $k \geq 0$, we take $X_{h}:=\mathcal{W}_{h} \times \mathcal{P}_{k}\left(\mathcal{T}_{h}, \mathbb{K}\right)$, with $\mathcal{W}_{h}:=\mathcal{P}_{k+1}\left(\mathcal{T}_{h}, \mathbb{M}\right) \cap$ $H(\operatorname{div}, \Omega, \mathbb{M})$ and here again $K_{h}=\mathcal{W}_{h}^{0} \times \mathcal{P}_{k}\left(\mathcal{T}_{h}, \mathbb{K}\right) \subset K$. It follows easily from Lemma 6.3 that Assumption 4 holds true.

Proposition 6.4 The spectrum of $T_{h}$ consists of $m:=\operatorname{dim}\left(X_{h}\right)$ eigenvalues, repeated accordingly to their respective multiplicities. It holds $\operatorname{sp}\left(T_{h}\right)=\{1\} \cup\left\{\eta_{h k}\right\}_{k=1}^{m_{0}}$, with $m_{0}=m-\operatorname{dim}\left(K_{h}\right)$. The eigenspace associated to $\eta_{h}=1$ is $K_{h}$. The eigenvalues $\eta_{h k} \notin\{0,1\}$ are non-defective and the corresponding eigenspaces lie in $K_{h}^{\perp_{B}}$. Moreover, $\eta_{h}=0$ is an eigenvalue with eigenspace $\operatorname{ker}\left(T_{h}\right)$.

Remark 6.2 It is straightforward that $\operatorname{ker}\left(T_{h}\right)=\left\{q_{h} I ; q_{h} \in V_{h}\right\} \times\{0\}$ where the space $V_{h}:=\mathcal{P}_{k+1}\left(\mathcal{T}_{h}\right) \cap H^{1}(\Omega) \cap L_{0}^{2}(\Omega)$ of continuous and piecewise polynomial functions of degree $\leq k+1$.

We define $\Xi_{h}: X \rightarrow X_{h}$ as in Lemma 6.4, which ensures Assumption 5 and guaranties by the way that the Galerkin method (3.1) provides a correct spectral approximation of the stress formulation of the Stokes eigenproblem (2.1) in the sense of Theorem 4.1. Moreover, Assumption 6 is satisfied thanks to (6.21). We can then rely on Theorem 5.2 to obtain the following rates of convergence for the eigenfunctions and eigenvalues.

Theorem 6.3 Let $\eta \notin\{0,1\}$ be an eigenvalue of $T$ of algebraic multiplicity $m$ and let $\mathcal{E}_{\eta}(X)$ be the corresponding eigenspace. There exists $h_{0}>0$ such that for all $h \leq h_{0}, T_{h}$ admits eigenvalues $\eta_{i, h}, i=1, \ldots, m$, repeated according to their respective multiplicity, such that

$$
\lim _{h \rightarrow 0} \widehat{\delta}\left(\mathcal{E}_{\eta}(X), \mathcal{E}_{\eta, h}\left(X_{h}\right)\right)=0 \text { and } \lim _{h \rightarrow 0} \max _{1 \leq i \leq m}\left|\eta-\eta_{i, h}\right|=0
$$

where $\mathcal{E}_{\eta, h}\left(X_{h}\right)$ is the $T_{h}$-invariant subspace of $X_{h}$ spanned by the eigenspaces of $\left\{\eta_{i, h}, i=\right.$ $1, \ldots, m\}$. Moreover, under the piecewise regularity assumption

$\mathcal{E}_{\eta}(X) \subset\left\{(\boldsymbol{\tau}, \boldsymbol{s}) \in H^{r}\left(\cup_{j=1}^{J} \Omega_{j}, \mathbb{M}\right) \times H^{r}\left(\cup_{j=1}^{J} \Omega_{j}, \mathbb{K}\right) ; \operatorname{div} \boldsymbol{\tau} \in H^{r}\left(\cup_{j=1}^{J} \Omega_{j}, \mathbb{R}^{d}\right)\right\}, \quad r>1 / 2$,

for $h$ small enough,

$$
\widehat{\delta}\left(\mathcal{E}_{\eta}(X), \mathcal{E}_{\eta, h}\left(X_{h}\right)\right) \lesssim h^{\min \{r, k+1\}} \text { and } \max _{1 \leq i \leq m}\left|\kappa-\kappa_{i, h}\right| \lesssim\left(h^{\min \{r, k+1\}}\right)^{2} .
$$




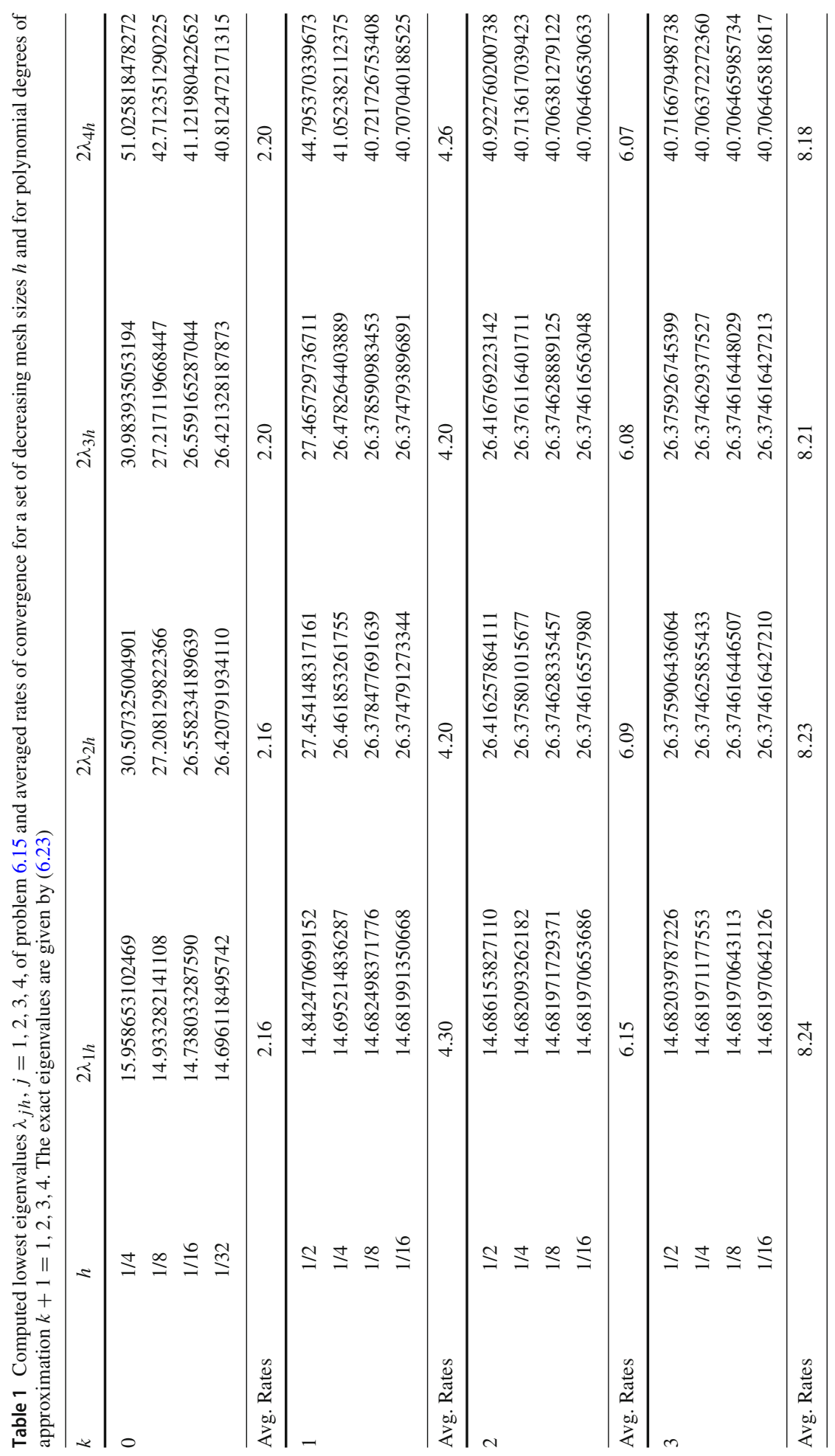


Remark 6.3 The analysis given in this section can be adapted to deal with Dirichlet-Neumann boundary conditions for eigenproblems (6.1) and (6.15) by defining the space $X$ as in [26] and by employing the quasi-interpolation operator with partial boundary conditions recently introduced by Licht in [25, Theorem 6.3].

\subsubsection{Numerical results}

On the unit disk, the eigenvalues of the Stokes eigenproblem (6.15) are given by the sequence $\left\{\frac{1}{2} J_{n \ell}^{2}\right\}_{n \geq 1, \ell \geq 1}$, where $J_{n k}$ is the $\ell$-th positive zero of the Bessel function $J_{n}$ of the first kind of order $n$. Accurate approximations of the first 4 eigenvalues are given by

$$
\begin{aligned}
2 \lambda_{1} & =J_{11}^{2} \simeq 14.681970642124 \\
2 \lambda_{2}=2 \lambda_{3}=J_{21}^{2} & \simeq 26.374616427163 \\
2 \lambda_{4}=J_{31}^{2} & \simeq 40.706465818200 .
\end{aligned}
$$

We use the open-source finite element software Netgen/NGSolve [30] to implement the Galerkin method (3.1) of the Stokes eigenproblem (6.15). We take advantage of the Netgen/NGSolve support for curved mixed finite elements of arbitrary order to base the construction of $X_{h}$ on an $H(d i v)$-conforming BDM-parametric element associated to an exact triangulation $\mathcal{T}_{h}$ of the unit disk. We notice that this leads to a conforming finite element approximation $X_{h}$ of $X$.

We denote by $\lambda_{j h}$ the approximation of $\lambda_{j}$ computed by solving problem 3.1 with $A$ and $B$ given by (6.17). We introduce the experimental rates of convergence

$$
r_{h}^{j}:=\frac{\log \left(\left|\lambda_{j}-\lambda_{j h}\right| /\left|\lambda_{j}-\lambda_{j \hat{h}}\right|\right)}{\log (h / \hat{h})}, \quad j=1, \ldots, 4,
$$

where $h$ and $\hat{h}$ are two consecutive mesh sizes.

We present in Table 1 the first four eigenvalues computed on a series of exact partitions of the unit disk $\bar{\Omega}$ with decreasing mesh sizes $h$, and for polynomial degrees $k+1=1,2,3,4$. We also report in the same table the arithmetic mean of the experimental rates of convergence obtained for each eigenvalue via (6.24). We observe that a convergence of order $2(k+1)$ is attained for each eigenvalue, as predicted by the error estimate given in (6.22).

Acknowledgements This work was funded by Ministerio Ciencia e Innovación Gobierno de España (Grant no. PID2020-116287GB-I00.

Open Access This article is licensed under a Creative Commons Attribution 4.0 International License, which permits use, sharing, adaptation, distribution and reproduction in any medium or format, as long as you give appropriate credit to the original author(s) and the source, provide a link to the Creative Commons licence, and indicate if changes were made. The images or other third party material in this article are included in the article's Creative Commons licence, unless indicated otherwise in a credit line to the material. If material is not included in the article's Creative Commons licence and your intended use is not permitted by statutory regulation or exceeds the permitted use, you will need to obtain permission directly from the copyright holder. To view a copy of this licence, visit http://creativecommons.org/licenses/by/4.0/.

\section{References}

1. Adams, S., Cockburn, B.: A mixed finite element method for elasticity in three dimensions. J. Sci. Comput. 25(3), 515-521 (2005) 
2. Amara, M., Thomas, J.M.: Equilibrium finite elements for the linear elastic problem. Numer. Math. 33(4), 367-383 (1979)

3. Arnold, D.N., Winther, R.: Mixed finite elements for elasticity. Numer. Math. 92(3), 401-419 (2002)

4. Arnold, D.N., Brezzi, F., Douglas, J., Douglas, J., Jr.: PEERS: a new mixed finite element for plane elasticity. Jpn. J. Appl. Math. 1(2), 347-367 (1984)

5. Arnold, D.N., Falk, R.S., Winther, R.: Mixed finite element methods for linear elasticity with weakly imposed symmetry. Math. Comp. 76(260), 1699-1723 (2007)

6. Arnold, D.N., Awanou, G., Winther, R.: Finite elements for symmetric tensors in three dimensions. Math. Comp. 77(263), 1229-1251 (2008)

7. Arnold, D.N., Awanou, G., Winther, R.: Nonconforming tetrahedral mixed finite elements for elasticity. Math. Models Methods Appl. Sci. 24(4), 783-796 (2014)

8. Boffi, D.: Finite element approximation of eigenvalue problems. Acta Numer. 19, 1-120 (2010)

9. Boffi, D., Brezzi, F., Gastaldi, L.: On the problem of spurious eigenvalues in the approximation of linear elliptic problems in mixed form. Math. Comp. 69(229), 121-140 (2000)

10. Boffi, D., Brezzi, F., Fortin, M.: Reduced symmetry elements in linear elasticity. Commun. Pure Appl. Anal. 8(1), 95-121 (2009)

11. Boffi, D., Brezzi, F., Fortin, M.: Mixed finite element methods and applications. In: Springer Series in Computational Mathematics, vol. 44. Springer, Heidelberg (2013)

12. Brezzi, F., Douglas, J., Jr., Marini, L.D.: Two families of mixed finite elements for second order elliptic problems. Numer. Math. 47(2), 217-235 (1985)

13. Christiansen, S.H., Winther, R.: Smoothed projections in finite element exterior calculus. Math. Comp. 77(262), 813-829 (2008)

14. Cockburn, B., Gopalakrishnan, J., Guzmán, J.: A new elasticity element made for enforcing weak stress symmetry. Math. Comp. 79(271), 1331-1349 (2010)

15. Dauge, M.: Elliptic boundary value problems on corner domains. In: Lecture Notes in Mathematics, vol. 1341. Smoothness and asymptotics of solutions. Springer-Verlag, Berlin (1988)

16. Descloux, J., Nassif, N., Rappaz, J.: On spectral approximation. I. The problem of convergence. RAIRO Anal. Numér. 12(2), 97-112, iii (1978a)

17. Descloux, J., Nassif, N., Rappaz, J.: On spectral approximation. II. Error estimates for the Galerkin method. RAIRO Anal. Numér. 12(2), 113-119, iii (1978b)

18. Ern, A., Guermond, J.-L.: Theory and practice of finite elements. In: Applied Mathematical Sciences, vol. 159. Springer-Verlag, New York (2004)

19. Ern, A., Guermond, J.-L.: Mollification in strongly Lipschitz domains with application to continuous and discrete de Rham complexes. Comput. Methods Appl. Math. 16(1), 51-75 (2016)

20. Gopalakrishnan, J., Guzmán, J.: Symmetric nonconforming mixed finite elements for linear elasticity. SIAM J. Numer. Anal. 49(4), 1504-1520 (2011)

21. Gopalakrishnan, J., Guzmán, J.: A second elasticity element using the matrix bubble. IMA J. Numer. Anal. 32(1), 352-372 (2012)

22. Grisvard, P.: Problèmes aux limites dans les polygones. Mode d'emploi. EDF Bull. Direction Études Rech. Sér. C Math. Inform. (1), 3, 21-59 (1986)

23. Jun, H.: Finite element approximations of symmetric tensors on simplicial grids in $\mathbb{R}^{n}$ : the higher order case. J. Comput. Math. 33(3), 283-296 (2015)

24. Kato, T.: Perturbation theory for linear operators. In: Classics in Mathematics. Springer-Verlag, Berlin (1995). Reprint of the 1980 edition

25. Licht, M.W.: Smoothed projections and mixed boundary conditions. Math. Comp. 88(316), 607-635 (2019)

26. Meddahi, S., Mora, D., Rodríguez, R.: Finite element spectral analysis for the mixed formulation of the elasticity equations. SIAM J. Numer. Anal. 51(2), 1041-1063 (2013)

27. Meddahi, S., Mora, D., Rodríguez, R.: Finite element analysis for a pressure-stress formulation of a fluid-structure interaction spectral problem. Comput. Math. Appl. 68(12, part A), 1733-1750 (2014)

28. Meddahi, S., Mora, D., Rodríguez, R.: A finite element analysis of a pseudostress formulation for the Stokes eigenvalue problem. IMA J. Numer. Anal. 35(2), 749-766 (2015)

29. Mercier, B., Osborn, J., Rappaz, J., Raviart, P.-A.: Eigenvalue approximation by mixed and hybrid methods. Math. Comp. 36(154), 427-453 (1981)

30. Netgen/NGSolve. Finite element library. https://ngsolve.org

31. Stenberg, R.: A family of mixed finite elements for the elasticity problem. Numer. Math. 53(5), 513-538 (1988) 
32. Shuonan, W., Gong, S., Jinchao, X.: Interior penalty mixed finite element methods of any order in any dimension for linear elasticity with strongly symmetric stress tensor. Math. Models Methods Appl. Sci. 27(14), 2711-2743 (2017)

Publisher's Note Springer Nature remains neutral with regard to jurisdictional claims in published maps and institutional affiliations. 